\title{
Cultural group selection, coevolutionary processes and large-scale cooperation
}

\author{
Joseph Henrich* \\ Department of Anthropology, Emory University, Geosciences Building, Atlanta, GA 30322-1720, USA
}

Received 17 January 2001; received in revised form 10 February 2003; accepted 24 April 2003

\begin{abstract}
In constructing improved models of human behavior, both experimental and behavioral economists have increasingly turned to evolutionary theory for insights into human psychology and preferences. Unfortunately, the existing genetic evolutionary approaches can explain neither the degree of prosociality (altruism and altruistic punishment) observed in humans, nor the patterns of variation in these behaviors across different behavioral domains and social groups. Ongoing misunderstandings about why certain models work, what they predict, and what the place is of "group selection" in evolutionary theory have hampered the use of insights from biology and anthropology. This paper clarifies some of these issues and proposes an approach to the evolution of prosociality rooted in the interaction between cultural and genetic transmission. I explain how, in contrast to non-cultural species, the details of our evolved cultural learning capacities (e.g., imitative abilities) create the conditions for the cultural evolution of prosociality. By producing multiple behavioral equilibria, including group-beneficial equilibria, cultural evolution endogenously generates a mechanism of equilibrium selection that can favor prosociality. Finally, in the novel social environments left in the wake of these cultural evolutionary processes, natural selection is likely to favor prosocial genes that would not be expected in a purely genetic approach.
\end{abstract}

(C) 2003 Published by Elsevier B.V.

JEL classification: D00; $\mathrm{C} 72$

Keywords: Cooperation; Altruism; Group selection; Coevolution; Dual inheritance theory

\section{Introduction}

As the experimental evidence for non-selfish preferences continues to mount in empirical work (Fehr and Gächter, 2000; Henrich et al., 2001; Bolton et al., 1998; Kagel and Roth,

\footnotetext{
* Tel.: +1-404-727-5248; fax: +1-404-727-2860.

E-mail address: jhenric@emory.edu (J. Henrich).
} 
1995), an increasing number of economists are turning to evolutionary biology and biological anthropology in search of plausible theoretical foundations (e.g. McCabe et al., 2000; Fehr and Gächter, 2002; Fehr et al., 2002; Gintis et al., 2001; Gintis, 2000; Ben-Ner and Putterman, 2000). Of particular interest to economic theorists is the evolution of prosocial preferences (e.g., tastes for altruism and altruistic punishment) that are capable of explaining cooperation and fairness in large groups and among unrelated strangers in non-repeated contexts. Unfortunately, the interdisciplinary nature and complexity of the literature on cooperation and altruism has led to some confusion about: (1) what constitutes an evolutionary 'solution' to the puzzle of prosociality, and (2) the process and potential importance of 'group selection'. In this paper, I will first layout a general framework for understanding evolutionary approaches to prosociality. Then, using this framework, I will critique and clarify some of the most common approaches to show how they fail to explain the prosocial preferences that promote large-scale cooperation among non-relatives without repeated interaction. Building on this foundation, the second half of the paper presents an alternative route to explain prosociality via culture-gene coevolution that keys on three things: (1) the difference between cultural transmission and genetic transmission, (2) the differences between genetic group selection and cultural group selection, and (3) the evolutionary interaction between genes and culture.

\section{The underlying structure of prosociality}

All genetic evolutionary explanations to the altruism dilemma are successful to the degree that they allow natural selection to operate on statistically reliable patterns or regularities in the environment. All too often, the assumptions or constraints that maintain this regularity are submerged in the basic structure or setup of the model — and not explicitly analyzed. To expose the required regularity, I develop a very simple model of cooperation. We begin with a population of $N$ individuals indexed by $i$ and subdivided into a number of smaller groups labeled with $j$. Each individual possesses either an altruistic gene or an egoistic gene tracked by $x_{i}$. When $x_{i}=1$ individual $i$ possesses the altruistic allele (version of the gene), and $x_{i}=0$ marks $i$ as possessing the egoistic allele. To derive the conditions for the spread of an altruistic gene, we will use the famous Price equation (Price, 1970, 1972):

$$
\bar{w} \Delta \bar{x}=\operatorname{Cov}\left(w_{i}, x_{i}\right)+E\left(w_{i} \Delta x_{i}\right)
$$

The Price equation is a simple statistical statement that relates the expected change in the frequency of a gene $(\Delta \bar{x})$ per generation, the absolute fitness $\left(w_{i}\right.$ : the number of offspring of $i)$, the average fitness of the population $(\bar{w})$, and the current frequency of the gene $\left(x_{i}\right)$. The $\operatorname{Cov}\left(w_{i}, x_{i}\right)$ term gives the covariance between absolute fitness and gene frequency, and $E\left(w_{i} \Delta x_{i}\right)$ represents the expected value of the within-individual change in $x_{i}$ (e.g., mutation) weighted by the individual's absolute fitness. Following Hamilton (1975), I am using the non-standard notational convention of leaving the subscripts on the variables inside of the covariance and expectation operators. This practice is required to maintain notational clarity when things get more complicated below. ${ }^{1}$ Eq. (1) provides a very general statement

\footnotetext{
${ }^{1}$ For example, using a more standard notational convention, one could write $\operatorname{Cov}(w, x)$ in lieu of $\operatorname{Cov}\left(w_{i}, x_{i}\right)$.
} 
about any evolutionary system. Later, I will derive and extend its application to cultural evolution and structured populations.

In this model, altruists bestow benefits on other members of their local group $j$ at a cost to themselves. Egoists do not bestow benefits on others, but both egoistic and altruistic group members will benefit from being in groups with many altruists. To investigate this, I specify $w_{i}$ as follows:

$$
w_{i}=\alpha+\beta_{w_{i} x_{i} \cdot x_{j}} x_{i}+\beta_{w_{i} x_{j} \cdot x_{i}} x_{j}+\varepsilon_{i}
$$

Here $i$ 's fitness is jointly determined by the effect of her genotype on her fitness, holding her local group composition constant, $\beta_{w_{i} x_{i} \cdot x_{j}} x_{i}$ ( $x_{j}$ is the average value of $x_{i}$ in group $j$ ), and, the effect of $i$ 's local group on her fitness holding the individual's genotype constant $\beta_{w_{i} x_{j} \cdot x_{i}} x_{j}$. The constant baseline fitness is $\alpha$ and $\varepsilon_{i}$ is the uncorrelated error term.

With Eqs. (1) and (2) we can derive a general statement about the conditions for the genetic evolution of altruism. Because we are interested only in the effects of natural selection, I will ignore non-selective forces such as recombination, drift, meiotic drive and mutation. This means $\Delta x_{i}=0$, so the expectation term drops out of (1). Substituting (2) into (1) yields

$$
\bar{w} \Delta \bar{x}=\operatorname{Cov}\left(\alpha, x_{i}\right)+\beta_{w_{i} x_{i}} \operatorname{Var}(x)+\beta_{w_{i} x_{j}} \beta_{x_{j} x_{i}} \operatorname{Var}(x)+\operatorname{Cov}\left(\varepsilon_{i}, x_{i}\right)
$$

By definition $\operatorname{Cov}\left(\alpha, x_{i}\right)$ and $\operatorname{Cov}\left(\varepsilon_{i}, x_{i}\right)$ are zero. Setting $\Delta \bar{x}>0$ gives the conditions for an increase in the frequency of the altruistic gene in the population (assuming both alleles exist in the population):

$$
\beta_{w_{i} x_{i}}+\beta_{w_{i} x_{j}} \beta_{x_{j} x_{i}}>0
$$

Eq. (4) shows that all solutions to the evolution of altruism-whether they are based on kinship, reciprocity or group selection (more on those later) — are successful according to the degree in which 'being an altruist' predicts that one's partners or group members are also altruistic (Frank, 1998). By definition $\beta_{w_{i} x_{i}}$ is negative because, ceteris paribus, altruists have lower fitness than egoists; $\beta_{w_{i} x_{j}}$ is always positive because, independent of one's own genotype, it is always better to be in a group with more altruists. This leaves $\beta_{x_{j} x_{i}}$, which captures the degree to which 'being an altruist' predicts 'being in an altruistic group'. If groups are randomly remixed every generation, $\beta_{x_{j} x_{i}}=0$, and altruism (even kin-based altruism) cannot evolve. However, if (for whatever reason) altruists can preferentially group with other altruists, $\beta_{x_{j} x_{i}}$ will be a positive number between zero and one, and altruism at least has a chance. If egoists can preferentially enter groups with altruists, $\beta_{x_{j} x_{i}}$ will be negative. Thus, altruistic genes can only spread if $\beta_{x_{j} x_{i}}$ is sufficiently close to one such that the benefits to being in an altruistic group outweigh the costs of bestowing benefits on others. Those with any familiarity with evolutionary theory may recognize (4) as a generalized version of "Hamilton's Rule" (Queller, 1992b). Expressing this in the standard notation, $\beta_{w_{i} x_{j}}$ is $\mathrm{B}$, the fitness benefits provided by altruists; $\beta_{w_{i} x_{i}}$ is $-\mathrm{C}$, the cost to the altruist; and $\beta_{x_{j} x_{i}}$ is $r$, which in the specific case of kin selection is interpreted as the coefficient of relatedness by descent from a recent ancestor. In general, however, $\beta_{x_{j} x_{i}}$ is not a measure of relatedness by descent from a recent common ancestor-although, as I will explain, that is one way to get a positive value of $\beta_{x_{j} x_{i}}$. Hereafter, for simplicity, I will refer to $\beta_{x_{j} x_{i}}$ as $\beta$. 
Most importantly, Eq. (4) shows that understanding particular solutions to the evolution of altruism requires analyzing how the model maintains a sufficiently high value of $\beta$. Thus, the acceptability or legitimacy of a theoretical solution depends on an evaluation of the constraints (or "special assumptions") that give rise to, and maintain, the statistical association $(\beta)$. Remember, however, that the greater the value of $\beta$, the greater the amount of altruism that can evolve and the greater the selective pressures for mutant genes that can 'beat the system' by exploiting $\beta$. Under different circumstances, in different species, different kinds of constraints or special assumptions may be warranted. The details of an organism's social structure, physiology, genome, cognitive abilities, migration patterns or imitative abilities may support some hypotheses and undermine others. Of course, by emphasizing the species-specificity of certain constraints, I do not mean to suggest that each constraint is equally likely to be observed in a randomly selected species. Surely, some constraints are more frequently satisfied in nature than others; nevertheless, the rarely satisfied constraints may provide the most interesting forms of altruism. Unfortunately, despite the need to defend a particular constraint vis-à-vis the details of that species that might justify focusing on that constraint over others, many students of evolution and human behavior regard only kinship- and reciprocity-based models of altruism as legitimate solutions. In contrast, approaches based on quite plausible constraints that may be satisfied only in the human case, such as those based on cultural group selection and culture-gene interaction, are neither widely considered nor understood by non-specialists. However, before dealing with alternative approaches, I will illustrate what I mean by 'constraints', how they facilitate solutions to the altruism dilemma, how these are often submerged in evolutionary models, and why decisions about what constitutes an "acceptable solution" are often arbitrary.

\section{Constraints and evolutionary models of altruism}

\subsection{Green beards}

I will begin with the classic example of a solution to the altruism dilemma based on an 'illegitimate' constraint: the 'green beard solution' (Dawkins, 1976). Imagine a gene that causes its bearer to have a green beard and to cooperate with other green-bearded individuals. Because green-bearded cooperators focus their benefits only on other green-bearded cooperators, natural selection will strongly favor this altruistic gene because $\beta=1$ (unless they are so rare that fellow green beards never find one another). More generally, if altruists can recognize other altruists (with a better than random chance, $\beta>0$ ) and preferentially bestow benefits on them, then some amount of altruism can be favored by natural selection. In the situation, the statistical regularity $(\beta)$ exploited by natural selection is the correlation between green beards (the signal) and altruism. The problem with this solution is that the informational regularity arises from a genetic (or physiological) constraint on the power of mutation to create selfish egoists with green beards (green-bearded defectors). Such a non-altruistic gene will exploit the green beard-cooperator regularity $(\beta)$ and destroy it in the process. Egoists with green beards will invade until $\beta$ is driven to 0 . Consequently, this model works only if we allow a mutational constraint. 
Is such a constraint acceptable? Popular writers on evolution often scoff at the absurdity of such a constraint (Low, 2000; Dawkins, 1976, 1982; Ridley, 1993), despite the fact that the available empirical evidence is somewhat mixed. Keller et al. (1998) have shown empirically that an odor-based signal explains large-scale cooperation in the red fire ant. In humans, experimental work suggests that people have some ability to distinguish cooperators from defectors (Frank et al., 1993; Brown and Moore, 2002), although other work says they cannot (Ockenfels and Selten, 2000; Ekman, 1992; Ekman and O'Sullivan, 1991; Henrich and Smith, in press). In either case however, what should be unacceptable is the common practice of simply assuming that individuals know the strategies of other individuals and assort according, without providing any justification (theoretical or empirical) as to why this should be so (e.g., Wilson and Dugatkin, 1997). This is tantamount to assuming the answer.

The green beard problem can be addressed as a problem in costly signaling (Frank, 1988; Gintis et al., 2001). If it costs less for cooperators to cue their strategy than for non-cooperators to falsely cue, then cooperation can be favored. This occurs because such signals allow the benefits of cooperation to be preferentially delivered to other cooperators. From the perspective of Eq. (4), cooperation can evolve because this signaling allows for the non-random formation of groups such that cooperators find themselves grouped with other cooperators. Egoists cannot fake signals because it is too costly for them, so they find themselves with other defectors.

There are four problems with the costly signaling approach to green beard altruism. First, it relies on the constraint that it is cheaper for cooperators to send the cues of cooperation than for defectors. Mutations that allow defectors to send these signals for the same cost as altruists cannot be permitted-this is a key constraint that makes the whole thing work. Second, as noted above, empirical evidence for an ability to distinguish 'cooperators' from 'defectors' without extensive interaction is contradictory and ambiguous. The ability of good actors (e.g., in the movies) to evoke powerful emotions in us by pretending to be either a valiant 'cooperator' or a malevolent 'defector' suggests there is some variation in the ability to send false signals (so, why are not we all good actors?). Third, we need to explain cooperation and trust among anonymous people (who cannot see one another) in one-shot encounters such as we observe in experimental economic games (Kagel and Roth, 1995; Davis and Holt, 1993; Fehr et al., 2002). In most of these situations, there is no chance for signaling of any kind. So, at best, this approach solves only one kind of altruism problem. Fourth, if such costly signaling explains the degree and kind of cooperation in humans, why has not this mechanism generated much more cooperation in non-human animals, such as chimpanzees, elephants and dolphins?

\subsection{Kin-based selection}

Kin-selection models actually make use of a variety of different constraints. In some kin-based approaches, the nature of a species' social (or family) structure produces a whole host of possible statistical associations that can be exploited by natural selection. For example, if sisters tend to hang around their mom, genes that cause their bearer to cooperate with others 'hanging around mom' may spread because-if they are really your sistersthey have at least a 50 percent chance of having the same altruistic gene as you. 'Hanging 
around mom' merely provides one possible predictor of kinship, and indirectly, of altruism. Consequently, social structures that require individuals to interact with relatives more frequently than non-relatives provide a potentially valuable informational regularity. If 80 percent of those receiving benefits from an altruist are full siblings of the altruist and the rest are non-relatives, then $\beta$ is approximately 0.40 (assuming the frequency of altruists in the rest of the population is very small).

This social structure constraint is only exploitable if, by whatever means, the underlying non-random association can be maintained. If non-altruistic mutants, posing as fake-family members, can frequently slip into nests or family groupings and obtain the benefits, then kinship solutions will suffer the same problem as green beard models. Among animals lacking the requisite social structure (e.g., some snake species), we should not expect the corresponding forms of altruism (kin or otherwise). Furthermore, the social structure-that often creates the requisite informational regularity-is usually taken as a given, rather than being a product of evolution with its own costs and benefits. Models never endogenize the costs of maintaining a particular family or social structure in analyzing the evolution and maintenance of altruism.

Other kin-based solutions are founded on kin-recognition mechanisms (Fletcher and Michener, 1987) that allow individuals to probabilistically spot their kin and direct altruism towards them. Qualitatively, this solution is the same as the green beard solution, except that having many of the same genes, being reared together, and the potential existence of other evolutionary pressures favoring kin recognition (e.g., inbreeding avoidance) makes the explanation much more plausible - though not fundamentally different - than unjustified green beards. Combinations of kin-cues may make it quite costly for natural selection to produce faker-defectors.

Finally, the rules of inheritance that make kinship a valuable predictor of altruism, are often taken as given despite the fact that certain alleles can violate these rules (e.g., meiotic drive and gametic selection). A non-altruistic gene that is transmitted from parent to offspring with a greater than 50 percent chance (contrary to Mendelian rules) could spread and gradually corrode the informational regularity upon which some forms of kin altruism are based. ${ }^{2}$

Kin-based mechanisms should be designed to focus benefits only on close relatives, and thus kin selection does not help us to solve the problem of cooperation among large groups of unrelated individuals unless our kin-psychology is making a lot of big mistakes by confusing large numbers of non-relatives and strangers with close relatives. This version of the "big mistake hypothesis" (Boyd and Richerson, 2002a) suggests that, because our psychology supposedly evolved in small groups with high degrees of interrelatedness, kin selection

\footnotetext{
${ }^{2}$ Meiotic drive and gametic selection distort (relative to Mendelian rules) the frequencies of different alleles available for recombination. Using a meiotic drive example, imagine two alleles: a $\mathrm{C}$-allele (cooperator) and a D-allele (defector). Diploid individuals who are CC or DD produce all C-gametes and all D-gametes, respectively. When paired with C-alleles, however, D alleles use "chemical warfare" such that CD individuals produce, on-average, a proportion $1-k$ of C-gametes and $k$ of D-gametes $(k>0.5$, under Mendelian rules $k=0.5)$. This means that if I am CD and we are brothers, I would be mistaken to assume that you have a 50 percent chance of possessing a copy of my C-allele. Because of meiotic drive we would be less related vis-à-vis the relevant allele than brothers would normally be for other alleles (those not on the Y-chromosome). In mice, the t-allele is transmitted over 90 percent of the time to offspring $(k=0.90)$.
} 
(along with reciprocity, see next section) favored a psychology in humans that is designed to generously bestow benefits on members of their groups. According to this idea, natural selection apparently neglected to provide humans with the ability to distinguish kin and long-term reciprocators from anonymous strangers in ephemeral interactions. Thus, in the novel world of large-scale, complex societies, this once adaptive psychological propensity misfires, giving us large-scale cooperation (Tooby and Cosmides, 1989). Using this line of reasoning, a variety of scholars have attempted to explain why we observe altruism, trust, cooperation, fairness and punishment in one-shot experimental games (Hoffman et al., 1998; Ben-Ner and Putterman, 2000).

This explanation has a number of problems. First, even in small-scale societies, there are plenty of distant relatives, and probably a fair number of strangers, that altruists need to distinguish from close kin- $\beta$ (relatedness by descent for alleles) decreases geometrically as the circle of kin expands from sibs, to half-siblings, to first cousins, and so on. The idea that individuals in small-scale societies did not have ephemeral encounters with anonymous others is empirically groundless and represents a popular anthropological myth spread by those unfamiliar with the details of the ethnographic and ethno-historical record. Second, although large-scale cooperation is prevalent in many societies, people everywhere favor their kin over non-kin-showing that we can, and do, distinguish these behaviorally (Daly and Wilson, 1988; Sepher, 1983; Wolf, 1970; Westermarck, 1894). Third, lots of non-human primates also live in small-scale societies but show no generalized tendency to cooperate with all members of their group. When non-human primates are placed in larger, evolutionarily novel groups (e.g., zoos and research centers), cooperation does not expand to the enlarged group. It is hard to believe that non-human primates do not make these big mistakes, but humans do. Boyd and Richerson (2002a) further summarizes the evidence against this hypothesis.

\subsection{Reciprocity}

Reciprocity provides another well-studied solution to the altruism dilemma. In reciprocity-based models with repeated interactions (usually modeled as a repeated prisoner's dilemma), it has been frequently shown that strategies that both bestow benefits on individuals who have bestowed benefits on them in the past and withhold future benefits from strategies that fail to reciprocate can be favored by selection if they are sufficiently common (Axelrod, 1984; Axelrod and Hamilton, 1981; Trivers, 1971). Repeated interaction using these kinds of reciprocating strategies produces the requisite informational regularity. Repeated interactions among the same individuals allows past behavior to guide the flow of benefits preferentially to other reciprocators $(\beta)$, and more repetitions allow groups of reciprocators to increase their fitness benefits relative to groups of non-reciprocators $\left(\beta_{w_{i} x_{j}}\right)$.

However, making such models work requires a couple of constraints that often go unstated. First, most models analyze only two strategies at a time (e.g., Axelrod, 1984) and do not consider the presence of additional strategies (even one additional strategy) maintained at low frequency by mutation, immigration, or non-heritable environmental variation. It turns out that if the full range of other strategies is occasionally allowed to mutate into existence at low frequencies, then no strategy (pure or mixed), such as "tit-for-tat" (TFT) 
and "always defect" (ALLD), ${ }^{3}$ is evolutionarily stable (Boyd and Lorderbaum, 1987; Lorberbaum, 1994). For example, if the game is repeated a sufficient number of times, tit-for-two-tats (TF2T) can invade a population of mostly TFT if "suspicious tit-for-tat" (STFT) is present at low frequency. TF2T is like TFT but requires two consecutive defections before defecting, while STFT defects on the first round and then plays TFT. Similarly, TF2T can be maintained at high frequency with low frequencies of STFT and TFT, unless ALLD enters the fray at low frequency, in which case it is possible for STFT to invade and become common. Notably, in a population of mostly STFT, we will observe mostly defection. The point here is that whether or not reciprocating strategies can remain stable depends on exactly how the mutational spectrum is restricted (errors can take the place of mutations, but then one needs to know the probability distribution of errors). Curiously, proponents of reciprocity-based altruism criticize green beard explanations because they require restricting the mutational spectrum (e.g. Dawkins, 1976). ${ }^{4}$

Second, it is also important to realize that most theoretical models of reciprocity explore only 2-person interactions. Despite this, many scholars have falsely assumed that the qualitative aspects of the 2-person results can be generalized to $n$-person situations (e.g., Patton, 2000). However, Boyd and Richerson's (1988) analysis of an $n$-person repeated prisoner's dilemma shows that the results do not generalize for groups larger than about 10 individuals. They show that the threshold frequency of reciprocators necessary to maintain stable cooperation in an $n$-person group increases with group size by the power $1 / n$. They further show that combining kinship (relatedness by common descent) with reciprocity does not substantially improve the results, as it does in the 2-person case. Reciprocity, on its own, is unlikely to solve the problem of cooperation in large groups (also see Bendor and Mookherjee, 1987; Joshi, 1987).

The final implicit constraint in reciprocity models lies in the fact that individuals must be able to accurately recognize their partner(s) in repeated games. If there are mutants capable of posing as the individual(s) who bestowed benefits in the last turn, then the benefits of reciprocity evaporate (as $\beta \rightarrow 0$ ). Some might think that although this may be a problem for cooperation in cognitively limited animals, it is not a serious problem for big-brained humans. Perhaps it is simply too costly for natural selection to produce doppelgangers capable of fooling human cognition - a cognition presumably maintained for other evolutionary reasons (which puts the costs of that cognition outside the problem). However, to the contrary, this is a serious problem for the kind and degree of cooperation we observe in many societies. Consider the effects of increasing the group size from 2 to 2000 or of increasing the delay between the time you receive benefits and the time when your next chance to bestow them occurs. In large-scale societies, reciprocators would potentially need to keep track of the acts of hundreds or thousands of individuals for years. Further, greater cognitive capacities seem less likely to promote cooperation via reciprocity than to promote defection by producing more sophisticated deception and strategies of exploitation.

\footnotetext{
3 TFT cooperates on the first round and then plays whatever its partner played on the previous turn. ALLD always defects on every turn.

${ }^{4}$ If pure strategies sometimes make mistakes by cooperating when their strategy indicated defection, or defecting when they wanted to cooperate, some pure strategies can be evolutionarily stable. Stable pure strategies include both reciprocating and non-reciprocating strategies, but not TFT (Boyd, 1989; Lorberbaum et al., 2002). Interested readers should also see Hirshleifer and Martinez (1988).
} 
As noted above, some scholars have argued that the high levels of prosocial behavior that we observe in contemporary societies represent the "misfiring" of an evolved psychology geared for reciprocity in the small-scale societies of our evolutionary history (Alexander, 1974, 1987; Hamilton, 1975; Tooby and Cosmides, 1989). In large-scale complex societies, this psychology of reciprocity causes us to mistakenly cooperate when we should defect. This explanation is not plausible and suffers the same problems as the kin-selection version of the "big mistake hypothesis" discussed above.

\subsection{Indirect reciprocity}

By assuming that individuals know something of their partner's previous behavior with other individuals (their reputation or ranking), models of indirect reciprocity attempt to explain generalized cooperation by expanding on the idea of reciprocity (Alexander, 1987). Summarizing results from several different modeling approaches suggests that such a setup only favors cooperation when: (1) populations are small, (2) errors in perception of past behavior are rare, and (3) the number of repeated rounds with the same group are many (Leimar and Hammerstein, 2001; Nowak and Sigmund, 1998; Boyd and Richerson, 1989). ${ }^{5}$ Each of these can be understood vis-à-vis Eq. (4). Accurate perceptions and/or small populations guarantee that altruists can use past behavior to preferentially interact with other prosocial strategies, providing a large $\beta$. Repeated rounds with the same group mean the benefits experienced by groups with more altruists will be relatively larger than those experienced by groups with fewer altruists (yielding a larger $\beta_{w_{i} x_{j}}$ ). Of course, if a mutant defecting-strategy can generate an inflated reputational signal, perhaps by paying other individuals to lie about the mutant's cooperative tendencies, then the predictive value of reputation will corrode and cooperation will collapse. The only way to prevent the entry of such strategies into the mix is to restrict them by fiat. Such a constraint in the mutational spectrum is exactly what popular writers lampoon when they criticize the green beard model.

Nevertheless, even if we accept this constraint, indirect reciprocity still fails to explain large-scale cooperation and altruism in one-shot, anonymous situations. The amount of cooperation supported by indirect reciprocity declines exponentially with increasing group size (Nowak and Sigmund, 1998; Leimar and Hammerstein, 2001), ${ }^{6}$ so large-scale cooperation is not explained. And, if the reputational information is removed from the model or made less inaccurate, indirect reciprocity can only sustain cooperation in very small groups (Boyd and Richerson, 1989). While the empirical evidence indicates that a reputation for being prosocial makes one more likely to receive benefits (Wedekind and Milinski, 2000), it also shows that people behave prosocially in both experimental and real settings without any reputational information, even in large groups (Fehr et al., 2002; Ledyard, 1995; Henrich and Smith, in press). But, as with direct reciprocity and kinship, some evolutionary researchers maintain the "big mistake" hypothesis for indirect reciprocity (Nowak and

\footnotetext{
5 Nowak and Sigmund's (1998) conclusions about indirect reciprocity on the evolution of cooperation were shown to be overstated and their modeling techniques incomplete by Leimar and Hammerstein (2001).

6 This claim may not be clear from Nowak and Sigmund (1998), but see the caption of Fig. 3. The authors write, "the averages over time of the frequency of cooperative strategies (defined by $k \leq 0$ ) are 90, 47, and 18 percent for, respectively, $n=20,50$ and 100” (Nowak and Sigmund, 1998, p. 574).
} 
Sigmund, 1998). This suggests that humans cannot tell the difference between small groups of repeated interactants and large groups of strangers - a claim contradicted by experimental evidence (Fehr and Henrich, forthcoming).

\subsection{Punishment}

Many theorists have attempted to solve the problem of cooperation in large groups by incorporating punishment (e.g., McAdams, 1998; Hirshleifer and Rasmusen, 1989; Fudenberg and Maskin, 1986; Axelrod, 1986). If cooperators punish defectors, then cooperation can be favored. However, if punishment is costly for the punisher (as it must certainly be), then cooperators who do not punish can invade because they avoid both the costs of being punished (for not cooperating) and the costs of punishing defectors (they are 2 nd order free riders). If the private benefits derived from punishing are greater than the costs of administering it, punishment may initially increase, but cannot exceed a modest frequency (Boyd and Richerson, 1992). Using punishment, the problem of cooperation becomes one of how to maintain punishers in the population. One way to do this is to limit the mutational spectrum by eliminating strategies that cooperate, but do not punish (e.g., Axelrod, 1986; Axelrod and Dion, 1988; Gintis, 2000). How we evaluate this approach depends on how likely such a mutational constraint is in nature-which means, without further justification, such explanations are on a par with green beard approaches. Another way to solve the problem of punishment is to incorporate a recursive punishing strategy in which punishers punish individuals who do not cooperate and those who fail to punish individuals who do not punish (Boyd and Richerson, 1992). This solution is a kind of mathematical trick that eliminates the cost of punishment by spreading it out over an infinite space. Surely people cannot track defections through a nearly infinite set of stages. Below, I will discuss a culture-gene coevolutionary solution, based on punishment, for the evolution of one-shot, $n$-person, cooperation (Henrich and Boyd, 2001) that builds on a more-plausible informational constraint.

In considering efforts to explain human prosociality, including the above-described approaches to altruism, it is essential to ask why the solution does not apply to non-human animals. Kinship and direct reciprocity, for example, have been quite fruitful for understanding a variety of social behavior in non-human animals. Therefore, if a particular approach claims to explain large-scale and anonymous cooperation in humans, the approach must also either predict it in other species (specifying the conditions) or explain why the model is restricted only to humans. Given that we have not observed human levels of non-kin prosociality in other mammals, approaches should specify why they apply exclusively to humans. None of the standard evolutionary explanations deliver this.

\section{The logic of selection within- and between-groups}

Before examining the evolution of large-scale prosociality using a coevolutionary approach, it is important to understand how any evolutionary problem-and in particular the evolution of prosociality — can be viewed and described using the logic of multi-level selection. This perspective contrasts within-group selection, which acts against altruists who 
are exploited by free riders from their groups, to between-group selection that favors groups with more prosocial individuals because such groups can outcompete groups dominated by free riders.

Debates about the potential relevance of 'group selection' (or 'multi-level selection'), particularly in its application to human behavior and cooperation, continue despite a fair amount of agreement among those who have seriously explored the question (Wade, 1985; Frank, 1995; Maynard Smith, 1998; Bowles, 2001; Gintis, 2000; Soltis et al., 1995). Since the publication of George Williams' Adaptation and Natural Selection in 1966 (Williams, 1966), which quite appropriately laid waste to a particularly naïve form of group- or species-functionalism that had been prominent in biology and anthropology during the preceding decades, a whole generation of biologists and anthropologists learned to scorn any explanation that involves selection among groups or proposes 'group-functional' traits. However, only a few years after Williams' book, Price $(1970,1972)$ provided an elegant formalization that showed, among other things, how the force of natural selection acting on genes can be partitioned into 'group-level' and 'individual-level' components. Unfortunately, the insight derived from Price's simple demonstration did not spread very far outside of theoretical evolutionary biology and failed to impede the spread of the belief that group-selectionist-thinking is somehow logically flawed, wrong-headed, or merely wishful thinking. This untutored dismissal of group selection, particularly in regard to cultural evolution, has slowed progress in understanding a variety of evolutionary processes.

The way evolutionary scholars often talk about 'group selection' leads people to mistakenly think of it as a separate process, somehow fundamentally different from 'individual selection' or 'natural selection' (e.g., Low, 2000; Ridley, 1993; Dawkins, 1976). Genetic evolution, at least from one perspective, is about changes in the frequency of alternative alleles, not about the frequencies of individual organisms or groups of organisms. Taking that as given, it is both possible and sometimes useful to write down accounting systems that track the frequencies of these alleles by examining their effects on fitness from different points of reference. Useful approaches might involve tracking the fitness of alleles, individuals, families, social groups, genomes, chromosomes, and so on. To show this, I develop a multi-group form of the Price equation that partitions the forces of natural selection into within- and between-group components. With this analytical tool, we can clarify the conditions under which the between-group component may overpower the within-group component and favor group-beneficial alleles or cultural traits.

We start with a population of $N$ individuals subdivided into groups indexed by $j$, each with $n_{j}$ members. There are no restrictions on how the groups are composed, except that all groups must contain at least one individual. The variable $x_{j}$ gives the frequency of the trait/allele in subpopulation $j, x_{j}^{\prime}$ represents the same frequency in the next time period (or generation), and $\Delta \bar{x}$ expresses the average change in the frequency of the trait under investigation. Incorporating $q_{j}\left(=n_{j} / N\right)$, the proportion of the total population accounted for in group $j$, and $q_{j}^{\prime}$ (the same proportion in the next time step) means

$$
\Delta \bar{x}=\sum_{j} q_{j}^{\prime} x_{j}^{\prime}-\sum_{j} q_{j} x_{j}
$$


Noting that $\Delta x_{j}=x_{j}^{\prime}-x_{j}$ gives us

$$
\Delta \bar{x}=\sum_{j} q_{j}^{\prime}\left(x_{j}+\Delta x_{j}\right)-\sum_{j} q_{j} x_{j}
$$

We can relate $q_{j}$ and $q_{j}^{\prime}$ by comparing the fitness of group $j, w_{j}$, to the mean fitness across all groups: $q_{j}^{\prime}=q_{j} w_{j} / \bar{w}$. With this, we arrive at the following expression:

$$
\Delta \bar{x}=\sum_{j} \frac{q_{j} w_{j}}{\bar{w}}\left(x_{i}+\Delta x_{i}\right)-\sum_{j} q_{j} x_{j}=\sum_{j} q_{j} x_{j}\left(\frac{w_{j}}{\bar{w}}-1\right)+\sum_{j} q_{j}\left(\frac{w_{j}}{\bar{w}}\right) \Delta x_{j}
$$

Then, using the standard definitions of Covariance and Expectation, the above simplifies to

$$
\bar{w} \Delta \bar{x}=\operatorname{Cov}\left(w_{j}, x_{j}\right)+E\left(w_{j} \Delta x_{j}\right)
$$

As above, I used a non-standard notational practice and left the subscripts on the variables inside the operators to remind the reader that these are expectations taken over the groups indexed by $j$ and weighted by $q_{j}$.

This form of the Price equation is useful because it partitions the forces that influence the dynamics in an intuitively tractable fashion. The covariance term summarizes the effect of selection between groups-group competition. If $w_{j}$, the fitness of group $j$, positively covaries with the frequency of the trait in group $j\left(x_{j}\right)$, then this term will favor an increase in the average value of the trait in the overall population.

The second term, $E\left(w_{j} \Delta x_{j}\right)$, conceals the effect of selection within groups by intermingling it with the effects of transmission (i.e., mutation, non-random mating, etc.). We can further separate out the influence of selection within groups by re-applying the above technique to the term $w_{j} \Delta x_{j}$, which itself is the product of two average values: $w_{j}$ is the average fitness of individuals in group $j$, and $\Delta x_{j}$ is the average change in the frequency of the trait in group $j$. To do this, individuals within group $j$ are indexed by $i$. As above, $x_{i j}$ is the frequency of an allele (trait) in individual $i$ and takes on the values of 1 (present) or 0 (absent). Substituting this into (6) yields

$$
\bar{w} \Delta \bar{x}=\overbrace{\operatorname{Cov}\left(w_{j}, x_{j}\right)}^{\text {selection between groups }}+\overbrace{E_{j}\left(\operatorname{Cov}\left(w_{i j}, x_{i j}\right)+E\left(w_{i j} \Delta x_{i j}\right)\right)}^{\text {selection and transmission within groups }}
$$

To focus entirely on the effects of selection, we ignore any effects arising from the transmission process (e.g., recombination, mutation, etc.) and migration between groups (more on this below), so $\Delta x_{i j}=0$, and (7) simplifies to (8):

$$
\bar{w} \Delta \bar{x}=\underbrace{\beta_{w_{j}, x_{j}} \operatorname{Var}\left(x_{j}\right)}_{\text {selection between-groups }}+\underbrace{E\left(\beta_{w_{i j} x_{i j}} \operatorname{Var}\left(x_{i j}\right)\right)}_{\text {selection within-groups }}
$$

Eq. (8) tells us that the change in the frequency of an allele created by natural selection acting on individuals can be partitioned into between-group and within-group components. The magnitude of the between-group component depends on the amount of variation between groups and the size of the partial regression coefficient of group fitness on the frequency 
of the trait in the group. The sign of the between-group component depends only on the sign of the partial regression coefficient. If having a higher frequency of the trait predicts higher group fitness (e.g., more cooperation), then the between-group component is positive. Similarly, the magnitude of the within-group component depends on the variation within groups and on the partial regression coefficient of individual allele frequency on individual fitness within groups. The sign of the within-group component depends on the sign of the regression coefficient. Using partial regression coefficients reminds us that although fitness is certainly influenced by many factors (too many to consider here), what matters at any given time is the linear relationship between fitness and the trait value, holding all other factors constant. Often the within- and between-group components have the same sign, so the partitioning provides little insight. However, if $x$ happens to track the frequency of an altruistic allele, the within-group regression coefficients will be negative (by definition, altruists are exploited by non-altruists), while the between-group regression coefficient will be positive (groups with more altruists do better than groups with fewer). 'Group selection' or, more accurately, 'genetic group selection' occurs when the between-group component of natural selection acting on gene frequencies ${ }^{7}$ overcomes the within-group forces to favor an equilibrium different from that which would be favored by the within-group component acting alone. ${ }^{8}$

\subsection{The between-group component is often small relative to the within-group component}

The between-group component of selection shown in (8) may be important for understanding a wide range of evolutionary phenomena. However, the real controversy and confusion erupts in its application to the evolution of cooperation/altruism. This is because under a wide variety of conditions, the between-group term in (8) is often small (or rapidly becomes small) relative to the within-group term. This occurs because migration, group formation, and other kinds of genetic mixing among groups deplete the variation among groups $\left(\operatorname{Var}\left(x_{j}\right)\right)$ while increasing (or at least maintaining) the variation within groups, $\operatorname{Var}\left(x_{i j}\right)$. To see this, consider a population consisting of two equally sized groups, one initially composed entirely of kindly altruists, who bestow fitness benefits on all other members of their group, and the other of selfish egoists who do not bestow benefits. This condition makes the between-group favoring altruism as big as possible, $\operatorname{Var}\left(x_{j}\right)=0.25$, while making the within-group variation zero, $\operatorname{Var}\left(x_{i i}\right)=0$. According to Eq. (8), altruistic genes will initially spread, no matter what the relative difference (in magnitude) is between the regression coefficients, because the within-group term is zero. If we keep the groups isolated for a long time and assume no mutation, the population will eventually be dominated entirely by altruists. However, if we allow a small amount of migration between the populations,

\footnotetext{
${ }^{7}$ I specify 'acting on genes' because natural selection can act on any kind of heritable phenotypic variation. Both cultural transmission and the transmission of acquired immunities provide examples of non-genetic, heritable variation that could be subject to natural selection.

${ }^{8}$ Hamilton (1975) re-derived his famous expression (Eq. (4); Hamilton, 1964, 1972) using the logic of selection within and between groups more than a quarter of a century ago, but the theoretical implications of his demonstration are still not widely appreciated by students of evolution and human behavior (also see Queller, 1992b).
} 
say 5 percent exchanged per generation, the variation between groups, $\operatorname{Var}\left(x_{j}\right)$, will begin to decline and rapidly approach zero. At the same time, migration will drive the variation within-groups, $\operatorname{Var}\left(x_{i j}\right)$, towards its maximum value of 0.25 . Ignoring the fitness bounty reaped by immigrant egoists in the altruistic population, this migration rate alone will reverse the initial values of the between- and within-group variations in about 40 generations. Thus, a great deal of theoretical work shows that genetic group selection will only lead to substantial levels of altruism when groups are very small, migration rates are quite low, and the intensity of selection among groups is high compared to the intensity of selection within groups (Rogers, 1990; Crow and Aoki, 1982; Aoki, 1982; Boorman and Levitt, 1980; Kelly, 1992). In short, models for the evolution of altruism via genetic group selection are theoretically possible under the right constraints; however, most researchers do not expect these to be satisfied very often. With regard to humans, evidence from paleoanthropology and extant small-scale societies does not support such stringent constraints. On the contrary, the mating systems in many small-scale societies favor substantial genetic mixing between groups (Lee, 1979; Richerson and Boyd, 1998) that rapidly deplete $\operatorname{Var}\left(x_{j}\right)$. Confirming this deduction (which is based on ethnographic data), the available genetic evidence from small-scale societies shows only small amounts of variation across groups (Hartl and Clark, 1989, pp. 300-301; Brown and Armelagos, 2001).

\subsection{Multiple stable equilibria can resist the force of migration and maintain variation between groups}

It is important to realize that the evolutionary dynamics associated with many different forms of social interaction, putting cooperation dilemmas aside, do not suffer from the depletion of between-group variation described above, and thus the between-group component is much more likely to be important. This occurs when local interaction between individuals can result in multiple locally stable equilibria. Unlike 'group-selected' solutions in cooperative interactions, neither small group size nor low migration rates are necessary to maintain between-group variation because within-group selection can counteract the genetic mixing produced by migration. This allows between-group selection to favor groups at equilibria that produce the highest mean group fitness (the efficient equilibrium). Therefore, when populations are structured and social interactions produce multiple stable equilibria, the between-group component is likely to have an important influence on the final distribution of behaviors/strategies (Boyd and Richerson, 1990). Thus, contrary to popularized claims about the general unimportance of group selection for understanding social behavior (laying aside the specific issue of cooperation), understanding the between-group component may be essential to explaining a wide range of behavior, especially in highly social species. ${ }^{9}$ Simple game-theoretic examples of interactions with multiple equilibria include coordination interactions, battle-of-the-sexes games, mutualistic interactions (e.g., the 'stag hunt'

\footnotetext{
${ }^{9}$ Male vs. female exogamy provides an example of a social behavior with multiple equilibria. Assuming that there is a big cost to inbreeding and a polygynous mating system that produces many of unknown paternal half siblings, selection should favor one sex leaving the natal group. It is plausible that in many cases there are two stable equilibria: (1) males leave and (2) females leave. Both equilibria are found in nature.
} 
game, Hirshleifer, 1982) and some models of reciprocity (Axelrod and Hamilton, 1981; Boyd, 1988).

To illustrate, imagine two proto-human groups. Due to random variation, one social group possesses a small number of mutants capable of using gestures (hand signals, body positions and facial expressions) to communicate with other such mutants, while the other group contains a similar small number of mutants capable of using verbalizations to communicate with other mutant-verbalizers; however, gestural communicators cannot communicate with verbalizers, and vice versa. If increased communicative abilities, in any form, are favored by natural selection in both groups, then the relative strength of selection on a particular form of communication (gestural vs. verbal) depends, in part, on the frequency of other individuals in the group who are capable of the same form of communication. Natural selection acting within the first group favors all gestural communicators, while in the second group it favors all verbalizers. Within-group forces will drive the first group towards all-gesturers and the second group towards all-verbalizers. Unlike cooperative dilemmas, migration between groups, which would tend to deplete the variation between groups, will be opposed by within-group frequency-dependent selection. If this within-group selection is strong enough, the variation between groups will remain high. Although verbalizers cannot invade the gestural group because of the coordination problem, groups of verbalizers may have a number of advantages over groups of gestural communicators such as: (1) communication is possible in darkness, through walls, in dense forest, and around topographical features, (2) communication is possible while using the hands for something else, and (3) communicating while injured is easier. As a consequence, the mean fitness of the verbalizer-group may be higher than the gestural group. If so, in the long run verbalizer-genes will be favored over gestural genes because of genetic group selection (i.e., the between-group component). As we will see later, cultural processes are even more likely to generate multiple stable equilibria, so the between-group component of cultural evolution is even more likely to be an important force of selection among culturally evolved equilibria.

\subsection{Viewing kinship and reciprocity as group selection}

In my view, the best way to view 'kin selection', 'reciprocity' and 'group selection' is as historically derived labels for different types of constraints (or classes of special assumptions). Imposing these constraints generates opportunities for natural selection to favor certain kinds of altruism. Often the labels seem to depend mostly on how the problem was initially set up. For example, why not consider explanations of altruism based on kin-recognition as plausible forms of 'green beard selection'? ${ }^{10}$ Many students of evolution and human behavior do not realize that 'inclusive fitness' (which was first used to derive (4) using kinship; Hamilton, 1964), 'individual fitness' (first used to derive (4) for 'reciprocal altruism' by Axelrod and Hamilton, 1981), and 'group selection' (i.e., the partitioned Price equation was used to derive (4) in Price, 1972; Hamilton, 1975) are simply three systems of gene-tracking and fitness accounting from three different perspectives. Any solution can be reformulated from each perspective to yield the identical answer. Hamilton (1975), for

\footnotetext{
${ }^{10}$ Note that sexual recombination does not work against kin recognition in the same way that it does against non-kin green beard genes (unless they are on the Y-chromosome).
} 
example, reformats his kin-selection approach using the Price equation, instead of tracking inclusive fitness.

To demonstrate the relationship between the 'group selection' approach and reciprocity, I will analyze a standard, 2-person, reciprocity model using the partitioned Price equation (4). In doing this, we observe that reciprocity favors cooperation when the between-group component overpowers the individual component. Of course, it is equally possible to re-derive typical 'group selection' solutions (selective emigration, assortative interaction, etc.) using inclusive fitness, or even by tracking individual fitness. None of the accounting techniques can claim general superiority over the others (Frank, 1998; Queller, 1992a; Sober and Wilson, 1998; Wade, 1985). However, despite the fact that it is possible to do this, it is neither easy nor particularly desirable.

In the repeated prisoner's dilemma described here, individuals are paired in groups that play for $m$ rounds each generation. During each round, individuals play either 'cooperate' (C) or 'defect' (D). Table 1 gives the payoffs received by the row player. The index $j$ labels three groups 1, 2, 3. Each group consists of two individuals labeled $i=1$ or 2 . Group $j=1$ contains two individuals who both play the strategy tit-for-tat. Group $j=2$ contains one tit-for-tater and one defector (who always plays D). Group $j=3$ contains two defectors. The variable $x_{i j}$ equals 1 for tit-for-tater's and 0 for defectors. The variable $x_{j}$ represents the frequency of tit-for-tater's in group $j$. Eq. (8) gives the change in frequency of tit-for-tater's. Here are the components of (8):

$$
\begin{aligned}
& E\left(\beta_{w_{i j} x_{i j}} \operatorname{Var}\left(x_{i j}\right)\right)=\frac{1}{3}[(0)+(-4)(0.25)+(0)]=-0.33, \quad \operatorname{Var}\left(x_{j}\right)=0.167, \\
& \beta_{w_{j}, x_{j}}=m
\end{aligned}
$$

Substituting these into Eq. (8) gives us

$$
\bar{w} \Delta \bar{x}=\underbrace{0.167 m}_{\text {between-group }}-\underbrace{0.33}_{\text {within-group }}
$$

This equation shows that it is the between-group component of natural selection that favors the evolution of reciprocity (in this case tit-for-tat), not 'individual selection'. In fact, the within-group component is always negative-meaning 'individual selection' always selects against reciprocity. Within groups, tit-for-tater's never do better than a fitness tie (with another tit-for-tater) and always lose against defectors-thus the within-group regression coefficients, $\beta_{w_{i j}} x_{i j}$, are always zero or negative. The magnitude of the positive

\begin{tabular}{|c|c|c|}
\hline \multirow{2}{*}{$\begin{array}{l}\text { Player } 1 \\
\text { plays }\end{array}$} & \multicolumn{2}{|c|}{ Player 2 plays } \\
\hline & $\mathrm{C}$ & $\mathrm{D}$ \\
\hline $\mathrm{C}$ & 2 & -1 \\
\hline $\mathrm{D}$ & 3 & 0 \\
\hline
\end{tabular}

Table 1

Two-person prisoner's dilemma payoff matrix ${ }^{\mathrm{a}}$

\footnotetext{
${ }^{\text {a }}$ Payoffs are for the row player (Player 1). Players 1 and 2 are interchangeable. ' $\mathrm{C}$ ' indicates cooperation and ' $\mathrm{D}$ ' indicates defection.
} 
between-group component (i.e., group selection) depends on $m$, the number of rounds of play. Assuming groups are randomly remixed every generation, $m$ controls the migration rate between groups. As $m$ increases, groups become more stable. When $m=1$, groups completely remix after every round and the between-group component is minimized. Reciprocity is favored when $m$ is sufficiently large that teams of reciprocators can run-up their combined fitness total sufficiently to overcome the relative fitness losses they suffer at the hands of defectors, within groups. The fact that the between-group component is what drives reciprocity is often lost because it is substantially easier to solve this problem using the 'individual fitness-accounting' approach — in which a strategy's relative fitness is calculated by averaging across all possible groups (for more on this, see Sober and Wilson).

\section{Kinds of cooperation and altruism}

We are not limited to picking only one solution to the altruism dilemma, especially in the human case. Our psychology may embody the effects of natural selection having taken advantage of different stable informational regularities over our evolutionary history. For example, the nature of the human family-with its division of labor and stability (Boyd and Silk, 1997; Klein, 1989)—suggests that human ancestors were likely to have repeatedly found themselves in small sub-groups (called 'families') in which other individuals were likely to have the same genes by descent from a recent ancestor. Consequently, like other primates, we are likely to have some cognitive machinery dedicated to bestowing benefits on kin. Similarly, life in small-scale, fairly stable, groups may provide for lots of repeated interaction with unrelated or distantly related individuals. Assuming our cognition/emotional system was complex enough that mutants could not easily pose as friends, then we should expect a psychology that favors helping well-known, reliable reciprocators (i.e., friends) — and for distinguishing them from others, especially anonymous strangers. Unfortunately, kin selection can only explain cooperation among close kin, and reciprocity (including indirect reciprocity) is limited to small groups with lots of repeated interaction and high quality reputation information. Experimental findings from many small- and large-scale societies show that people will trust, cooperate and behave altruistically toward anonymous individuals in simple one-shot games (Henrich et al., 2001; Roth et al., 1991; Fehr and Gächter, 2000, 2002; Batson and Shaw, 1991) — a finding further confirmed by lots of ethnographic evidence (Richerson and Boyd, 1998, 2000). As a consequence, none of the above solutions seems capable of: (1) explaining the large-scale cooperation among non-relatives that we observe in both modern and pre-modern societies, including foraging societies, ${ }^{11}$ or (2) isolating why evolutionary processes have only led to large-scale cooperation in our species; if all this prosociality is only a product of

\footnotetext{
11 Two things are worthy of note with regard to foraging societies. First, the nature of sharing, especially of sharing game, cannot be explained by either reciprocity- or kin-based theories (Bliege Bird and Bird, 1997), even in the smallest small-scale societies. Many of the simplest societies such as the !Kung, have quite strong institutions for maintaining cooperation on a scale much larger than the family or the band (Wiessner, 1983). Second, contrary to the view of foraging created by anthropologists studying extant groups, lots of archaeological and ethno-historical data indicates that foraging societies can be politically, economically and socially complex, with large-scale cooperation, social stratification and a substantial division of labor (Arnold, 1996; Roscoe, 2002).
} 
reciprocity and kinship, why do not Japanese macaques forced to live in large zoo populations develop armies, recycling, taxation, storage, and other aspects of human social organization?

\section{Cultural group selection, cultural transmission and large-scale cooperation}

In many social groups (but not all), humans cooperate on a larger scale than any other species-with the possible exception of eusocial insects. Interestingly, humans are also the most proficient at, and most reliant on, social learning to acquire behavioral practices and strategies. Our cognitive abilities to acquire information via imitation and other forms of direct social learning far exceed those of any other species (Tomasello, 1994, 2000). The operational details of these learning mechanisms appear to have been 'designed' by natural selection to extract useful information from the social world - that is, from the minds of other individuals (Boyd and Richerson, 1985; Henrich and Gil-White, 2001; Henrich and Boyd, 1998). Perhaps it is merely a coincidence that humans are both the most cooperative species and the most reliant on, and proficient at, social learning. However, I argue that the nature of our cultural transmission capacities, and of human psychology more generally, creates stable behavioral equilibria consisting of combinations of cooperation and punishment that are not available to genetic evolutionary processes in acultural species. The existence of these additional, culturally evolved, behavioral equilibria make the group selection component of cultural evolutionary processes much more powerful relative to the within-group component than can occur in genetic evolution. By systematically altering the social environment in favor of prosocial phenotypes, cultural processes create the conditions for natural selection to favor prosocial genes that could not otherwise be favored in mammalian social species because non-humans lack the requisite social learning capacities (e.g., high fidelity imitation) that give rise to gene-culture coevolutionary processes.

We have empirical reasons to believe that culturally transmitted ideas, beliefs and values (i.e., information) are important for understanding human cooperation. First, unlike other animals, the domains of cooperative behavior in humans vary from place to place and from group to group. In some societies, people may cooperate in fishing and house-building, but not warfare. In neighboring groups that inhabit the same physical environment, people may cooperate in warfare and fishing, but not house-building. Thus, unlike cooperation in eusocial insects and kin-based altruism in non-human primates, there is a tremendous amount of variation in the cooperative domains among human groups that is independent of differences in physical environments or local ecologies (Kelly, 1985; Henrich and Boyd, 1998). Second, unlike non-human animals, the scale of human cooperation varies from little cooperation outside the extended family (Johnson, 2003; Johnson and Earle, 1987) to massive cooperation on the level of nation states containing millions of individuals. This observation is confirmed by experimental data using one-shot Ultimatum Games that show big differences across human societies (Henrich et al., 2001). In contrast, the scale of cooperation in non-humans does not vary much among social groups within a species, only among species. Given these two kinds of variation within our species, cultural evolutionary processes seem much more likely than genetic processes to explain the patterns-especially 
given the modest genetic heterogeneity found among humans as a species (compared to other species such as chimpanzees) and the relatively recent (last 5000 years) rapid emergence of very large-scale cooperation.

\subsection{Cultural group selection}

Interestingly, 'group-functional' explanations for cooperation and other forms of groupbeneficial behavioral patterns have long been part of anthropology and sociology (Spencer, 1891; Rappaport, 1984; Harris, 1977; Vayda, 1971; Turner and Maryanski, 1979). However, by the end of the 1970s the anti-group selection movement had penetrated cultural anthropology and, by analogy with biological evolution, was used to argue that the individual was the relevant level of analysis in cultural evolution (Harris, 1979, pp. 60-61). The problem was that both biology and anthropology lacked a sufficiently clear understanding of the differences between genetic and cultural evolution to understand why the between-group processes that were unlikely to account for seemingly group-beneficial behavioral traits in genetic evolution could still operate effectively (even rapidly; Boyd and Richerson, 2002b) in cultural evolution. Below, I explain why. ${ }^{12}$

The Price equation derived above, with genetic evolution in mind, turns out to be a very general statement about any evolutionary system, which we will use to frame our thinking about cultural evolution. The 'altruist gene' $(x)$ that we focused on in the earlier derivation could be any characteristic of any evolving system, including the frequency of hydrogen atoms in a cluster of galaxies, or a quantitative phenotypic measure such as IQ, 'cooperativeness in group fishing ventures', managerial success, malaria resistance, or the variance in height of brothers. To discuss cultural evolution using the Price equation, I re-label the variables to avoid confusion. Replacing $x, \phi$ measures a quantitative phenotypic trait that can be influenced by cultural transmission. As a behavior, preference or strategy, $\phi_{i j}$ could measure individuals' willingness to die for their country (or tribe) in war, the amount of time or money an individual contributes to charity, or how much of his total 'catch' a hunter brings back to share in camp (as opposed to that portion he eats alone at the kill site). In this context, $f_{i j}$ replaces $w_{i j}$ and gives the cultural fitness for particular values of $\phi_{i j}$. Cultural fitness measures the degree to which a particular value of $\phi$, representing stuff stored in the head of individual $i$ in group $j$ at time $t$, affects its proportional representation in the population at time $t+1$. It may be thought of as replicability, transmissibility or simply influence-note that nowhere in this formulation there is a need for cultural stuff to 'replicate' or be defined as discrete units. ${ }^{13}$ The cultural fitness of $\phi_{i j}$ is jointly determined by the operational details of our social learning psychology and by how that psychology interfaces with the environment (Boyd and Richerson, 1985):

$$
\bar{f} \Delta \bar{\phi}=\underbrace{\beta_{f_{j}, \phi_{j}} \operatorname{Var}\left(\phi_{j}\right)}_{\text {selection between-groups }}+\underbrace{E\left(\beta_{f_{i j} \phi_{i j}} \operatorname{Var}\left(\phi_{i j}\right)\right)}_{\text {selection within-groups }}
$$

\footnotetext{
12 In Economics, cultural group selection arguments were foreshadowed in the work of Hayek $(1979,1988)$.

${ }^{13}$ For a discussion of the confusion created by cultural 'replicators', see Henrich and Boyd (2002) and Boyd and Richerson (2000).
} 


\subsection{Cultural transmission, human psychology and between-group variation}

As I discussed earlier, the main problem with 'group selection' is the maintenance of between-group variation in the face of migration or other forms of mixing. Unlike genetic evolution, several different mechanisms will act to reduce the within-group term in (9), $\operatorname{Var}\left(\phi_{i j}\right)$, while maintaining the between-group term, $\operatorname{Var}\left(\phi_{j}\right)$, even in the face of substantial migration. I will discuss four of these mechanisms. The first two are rooted in the details of how our cognition exploits the distribution of behaviors and ideas among members of a social group in order to 'decide' which of these traits to acquire. As I will show, both evolutionary modeling and empirical data support the existence of these cultural transmission mechanisms. The third and fourth mechanisms are not cultural transmission capacities, but rather psychological 'tastes' or preferences for: (1) avoiding behaviors that deviate from the common pattern and (2) punishing those who do not conform to the expected pattern. In my view, these two 'tastes' are probably either the products of purely cultural evolution (driven by cultural group selection), or coevolved products of genes responding to the novel social environments created by cultural group selection. Thus, they cannot be responsible for initiating the coevolutionary process that led to high levels of cooperation in humans. Nevertheless, once brought into existence by the first two mechanisms, they further catalyze the cultural group selection process by bolstering the forces that create and maintain differences between groups. When applied to social interaction occurring in single groups, these four mechanisms create equilibria that do not exist in genetic systems, including equilibria with high levels of cooperation and punishment in one-shot, $n$-person games (Henrich and Boyd, 2001). With the emergence of multiple equilibria, cultural group selection supplies a process that selects among alternative stable equilibria in favor of the ones best able to compete with other groups (Boyd and Richerson, 1990).

The first mechanism, conformist transmission, is a psychological propensity to preferentially copy high frequency behaviors. By biasing individuals in favor of copying common behaviors, preferences or behavioral strategies, this transmission bias tends to homogenize social groups. There are both theoretical and empirical reasons to believe that humans possess a tendency to copy the most common behavior. Theoretically, Henrich and Boyd (1998) have shown that genes favoring a heavy reliance on social learning and conformist transmission (copying the majority) can outcompete genes favoring individual learning in both spatially and temporally varying environments. This model predicts two important things: (1) that individuals should increase their reliance on social learning when individual (or environmental) information becomes less certain or as the difficulty of the problem increases, and (2) that individuals should rely on copying the majority (conformist transmission) under a wide range of conditions (also see Boyd and Richerson, 1985; Ellison and Fudenberg, 1993).

Independent experimental work in psychology supports both predictions, as well as a number of other predictions arising from this model. Psychologists studying conformity have shown that as a task's difficulty and financial incentives rise, individuals increase their reliance on imitation (vs. individual analysis) regardless of whether others will know how they behave (reducing any fear of social sanctions; see Baron et al., 1996; Insko et al., 1985). Furthermore, with real money on the line, other experiments show that individuals rely on copying the majority in social dilemmas, both when self-interest conflicts with 
the group-interest and when self-interested choices correspond to group-interested choices (Smith and Bell, 1994; Wit, 1999). Finally, Henrich (2001) shows that the slow take-offs and 'critical mass tipping-point' observed in many empirical studies of the diffusion of innovations are quite consistent with the effects of conformist transmission.

The presence of this adaptive bias on our social learning cognition means that, in the absence of unambiguous information from the environment or other decisive social learning stimuli (such as a prestigious individual, see the next mechanism), individuals will preferentially copy the most common ideas, beliefs, values and practices. Because new immigrants and the offspring of immigrants will preferentially adopt the common practices, conformist transmission can maintain group differences in a way that genetic transmission cannot-because offspring acquire their genes from their parents, not from the group. ${ }^{14}$ Consequently, as a byproduct of its evolved design, conformist transmission decreases the phenotypic variation among individuals within groups, thereby depleting the strength of within-group cultural forces. As stochastic forces such as cultural drift (sampling errors in transmission), biological shocks (e.g., plagues) and environmental disasters introduce random variation between groups, conformist transmission will act to maintain this group-level variation — variation that would otherwise be depleted by migration between groups, natural selection and payoff-biased forms of cultural transmission. Thus, by reducing within-group variation and increasing between group variation, conformist transmission provides the raw materials for cultural group selection. ${ }^{15}$

Other cultural transmission mechanisms can create the same effect through other means. If individuals possess a psychological bias to preferentially copy people who are both more successful (get higher payoffs) and similar to themselves in some marker trait such as language or dress, then under a wide variety of conditions (even with substantial migration rates), these cultural transmission mechanisms push the variation within groups towards zero (opposing the force of migration), while sustaining substantial amounts of variation among groups (McElreath et al., 2003; Boyd and Richerson, 1987).

There are both theoretical and empirical reasons to believe that people preferentially copy successful individuals and people like themselves. Recently, Gil-White and I have argued that, with the rise of imitation in the human lineage, natural selection favored cognitive abilities to rank potential models according to their payoffs and to preferentially imitate highly ranked models. Among the cues individuals use to rank potential models is the amount of prestige-deference an individual receives from other people. This deference acts as an honest signal of whom other individuals believe is highly successful or skilled because deference is 'paid' to such individuals in exchange for copying opportunities (high quality information). This rank-based copying bias, which we call prestige-biased transmission, allows individuals to shortcut environmental or trial-and-error learning processes, and leap directly to better-than-average skills by imitating successful or skilled cultural models.

\footnotetext{
14 This is consistent with a substantial amount of work in psychology and behavioral genetics showing that children do not acquire much, via social learning, from their parents (Harris, 1998; Plomin et al., 2000).

15 This process also provides an explanation of why humans have different 'cultures' and other animals do not. That is, conformist transmission provides one important reason why people in the same social group tend to believe the same things and why these beliefs persist over long periods. Without a conformist component to create 'cultural clumps', social learning models predict (incorrectly) that populations should be a smear of ideas, beliefs, values and behaviors, and that group differences should only reflect local environmental differences.
} 
Further, because the world is a noisy, uncertain place, and it is often not entirely clear why a particular individual acquires great prestige or success, humans have evolved the propensity to copy a wide-range of cultural traits from prestigious individuals, only some of which may actually relate to the individuals' success (Henrich and Gil-White, 2001).

A substantial amount of empirical work from psychology, economics and anthropology confirms a variety of predictions derived from the above theory (Henrich and Gil-White, 2001). However, most importantly for our purposes, these findings confirm that people preferentially imitate the ideas, opinions, beliefs, strategies, and behaviors of prestigious individuals across a wide range of domains (even in domains outside the expertise of the prestigious individual). For example, in a synthesis of the diffusion of innovations literature, Rogers (1995) has shown that the rate of spread of novel technologies and new economic practices into different social groups depends on how quickly prestigious, local "opinion leaders" adopt these innovations. In the laboratory, using a multi-round, market game with substantial incentives in which the results of each player's decisions were posted between sessions, experimental economists unexpectedly found that MBA students tended to mimic the decisions of successful players, even though rewards were distributed on a competitive basis. Allowing imitation also moved the group average substantially closer to the optimal decision predicted by Portfolio Theory (Kroll and Levy, 1992). In a different experiment, Offerman and Sonnemans (1998) showed that subjects making investment decisions tended to copy the beliefs of successful individuals (about the current environment), even when players clearly knew that these individuals had the same information about the current situation (also see Pingle, 1995; Pingle and Day, 1996).

When coupled with prestige-biased transmission, a self-similarity transmission bias makes good adaptive sense. Individuals should imitate successful others only as long as those individuals are not too different from the imitators themselves-otherwise imitated traits may be inapplicable to the copier's particular abilities and circumstances. Interestingly, as an emergent byproduct, this combination of cultural learning mechanisms decreases within-group variation while increasing between-group variation (Boyd and Richerson, 1987).

Following this line of reasoning, theoretical work suggests that individuals in social groups throughout human history needed to coordinate their beliefs, norms and expectations in order to make economic exchanges, marry and raise children. In theoretical work examining the interaction of genes and culture in solving these coordination problems, McElreath et al. (2003) show that natural selection will favor the evolution of a bias to cue off salient symbolic markers when they covary with individuals' underlying norms of interaction — so by using symbolic markers individuals avoid acquiring norms that would produce costly uncoordinated interactions. Although no one has empirically examined the relationship between imitation and ethnic markers, more general evidence for self-similarity learning biases comes from the diffusion of innovations literature (Rogers, 1995, p. 286), laboratory psychology (Rosekrans, 1967; Stotland and Dunn, 1962, 1963) and studies of child development (Harris, 1998).

A third mechanism, punishment of non-conformists or norm violators, acts to homogenize social groups. A great deal of ethnographic and experimental research suggests that people in many societies will inflict punishment on individuals who violate group norms of behavior (Sober and Wilson, 1998; Roth et al., 1991; Henrich et al., 2001; Fehr and Gächter, 2002). 
If violators of group norms receive costly punishments then either prestige-biased transmission or simply trial and error learning will reduce the variation between groups. Costly punishments will mean lower payoffs for norm violators, so that under prestige-biased transmission, the behaviors of norm violators are less likely to spread. The problem with this norm-based punishment, as I discussed earlier, is the difficulty in explaining how it could evolve in a purely genetic system of inheritance. If punishing norm violators is costly to the punisher, then punishing strategies are unlikely to evolve under natural selection. In the next section, I describe how punishing behaviors can be stabilized if humans have a very small amount of conformist transmission bias in their social learning psychology.

A fourth mechanism, normative conformity, arises because individuals want their behavior to match the common behavior in their social group. Normative conformity differs from conformist transmission in that individuals are not using the frequency of a behavior, belief or idea as an indirect indicator of its worth. Instead, normative conformity alters individuals' socially displayed behavior (without necessarily changing their minds) because they want their behavior to match the majority, not because they 'believe' the majority is probably doing the smart thing. A vast amount of experimental work beginning with Asch's famous studies (e.g., Asch, 1951), and including cross-cultural work (Furnham, 1984), show that normative conformity is a robust part of human psychology (Neto, 1995), at least in complex societies (nobody has done work among small-scale societies). Baron et al.'s (1996) experimental work attempted to differentiate normative conformity from the effects of conformist transmission. Their results strongly suggest that people (i.e., university students) have both conformist transmission and normative conformity components to their psychology. These two psychological processes probably evolved for separate reasons. While conformist transmission seems to have evolved genetically as a shortcut means of acquiring useful information, normative conformity is more likely a product of cultural evolution or culture-gene coevolution, and it may have evolved in response to the spread of punishing strategies, as a means to manipulatively cue one's 'similarity' to other group members in order to gain the advantages of in-group membership and avoid punishment. Also, note that normative conformity may result in conformist transmission. Under some circumstances, imitators may mistakenly infer the underlying preferences or goals in observing the compliant behavior of models (by assuming the models 'like' to perform certain behaviors), and thereby acquire both the outward behavior and the underlying supporting preferences that were not originally possessed by the models.

Individually, and in combination, these mechanisms increase the importance of cultural group selection by creating a myriad of additional stable equilibria for all kinds of cultural traits, ideas, beliefs, values and practices, including those that govern social and cooperative interactions. As I discussed earlier, the between-group component becomes important in circumstances with multiple stable equilibria because within-group selective processes, acting to push the system back to the locally stable equilibria, oppose the effects of migration that tend to reduce the variation between groups $\left(\operatorname{Var}\left(\phi_{j}\right)\right.$ in Eq. (9)). The nature of the mechanisms described above means that most of the time these stable equilibria will be monomorphic (everyone possesses the same cultural traits), thus placing $\operatorname{Var}\left(\phi_{i j}\right)$ near zero.

In thinking about how the difference between cultural and genetic evolution might affect the spread of prosociality, it is also important to keep two other differences in mind. First, cultural evolution is likely to proceed much more rapidly than genetic evolution because 
mechanisms such as prestige- and conformist-biased cultural transmission favor rapid transmission and can spread novel behaviors, ideas and practices among populations within a single generation (Boyd and Richerson, 1985, 2002b) —note that substantial evidence indicates that most cultural transmission is not vertical (i.e., not parent to offspring; Plomin et al.). Second, cultural transmission is likely to be more subject to drift and random variation than genetic transmission. The imitative skills of humans are good, qualitatively better than any other animals, but certainly worse than genetic replication. The combination of these two differences means that human groups under cultural evolution will drift into the domains of attraction of alternative equilibria more often than in genetic evolution. Thus, cultural group selection will "see" a greater variety of equilibria than genetic evolution over the same amount of time.

\subsection{Conformist transmission can stabilize cooperation by stabilizing punishment}

Before discussing cultural group selection, it is necessary to see how cultural transmission mechanisms can combine to create alternative stable prosocial equilibria. Henrich and Boyd (2001) have shown that if human social learning psychology contains both a transmission bias to copy successful individuals ('payoff-biased' or prestige-biased transmission) and a bias to copy high frequency behaviors (conformist transmission), and there are an arbitrary number of 'punishing levels', then highly cooperative equilibria can exist even if conformist transmission is only a weak component of human cultural transmission.

A tendency to copy high frequency behaviors can stabilize costly cooperative strategies without punishment, but only if this conformist transmission is quite strong compared to payoff-biased transmission. All other things being equal, payoff-biased transmission causes higher payoff variants to increase in frequency, and thus cooperation is not evolutionarily stable under plausible conditions because not-cooperating leads to higher individual relative payoffs (within groups) than cooperating. Thus, on its own, payoff-biased transmission suffers the same problem as natural selection in genetic evolution. However, if our social learning psychology contains a combination of conformist and prestige-biased transmission (as I have argued above), then, if cooperation becomes common, conformist transmission will oppose payoff-biased transmission and favor cooperation. When cooperation is not too costly, conformist transmission will maintain cooperative strategies in the population at high frequency. However, because both theory and evidence (Henrich, 2001) suggests that conformist transmission is relatively weak compared to payoff-biased transmission (and the costs of cooperation are probably substantial), it seems unlikely that conformist transmission will be able to maintain cooperation.

A quite different logic applies to the maintenance of punishment. Suppose that culturally transmitted punishing and cooperating strategies are both common and that being punished is sufficiently costly so that cooperators have higher payoffs than defectors. Rare invading 2nd order free riders who cooperate but do not punish will achieve higher payoffs than punishers because they avoid the costs of punishing. Because defection does not pay, the only defections will be due to rare mistakes, and thus the difference between the payoffs of punishers and 2 nd order free riders will be relatively small compared to that between 1 st order free riders and cooperators. Hence, conformist transmission is more likely to stabilize the punishment of non-cooperators than of cooperation itself. As we ascend to 
higher order punishing, the difference between the payoffs to punishing vs. non-punishing decreases geometrically towards zero because the occasions that require the administration of punishment become increasingly rare. Second order punishing is required only if someone erroneously fails to cooperate and then someone else erroneously fails to punish that mistake. For third order punishment, yet another failure to punish must occur. As the number of punishing stages $(i)$ increases, conformist transmission, no matter how weak, will at some stage overpower payoff-biased imitation and stabilize common $i$ th order punishment. Once punishment is stable at the $i$ th stage, payoffs will favor strategies that punish at the $i-1$ order, because common punishers at the $i$ th order will punish non-punishers at stage $i-1$. Stable punishment at stage $i-1$ order means payoffs at stage $i-2$ will favor punishing strategies, and so on down the cascade of punishment. Eventually, common 1st order punishers will stabilize cooperation/altruism at stage $0 .{ }^{16}$

The constraint that allows cooperation to arise and remain stable in this model is the costliness of information. From the gene's point of view, this is a maladaptive side-effect of conformist transmission. If there were genetic variability in the strength of conformist transmission $(\alpha)$ and cooperative dilemmas were the only problem humans faced, then conformist transmission might never have evolved. However, human social learning mechanisms were selected for their ability to efficiently acquire adaptive behaviors over a wide range of behavioral domains and environmental circumstances-from figuring out what foods to eat, to deciding what kind of person to marry-precisely because it is costly for individuals to determine the best behavior (Henrich and Boyd, 1998). Thus, we should expect conformist transmission to be important in cooperation as long as distinguishing cooperative dilemmas from other kinds of problems is difficult, costly or error-prone. Looking across human societies we find that cooperative dilemmas come in an immense variety of forms, including harvest rituals among agriculturalists, barbasco (communal) fishing among Amazonian peoples, warfare, irrigation projects, taxes, voting, meat sharing and anti-smoking pressure in public places. It is difficult to imagine a cognitive mechanism capable of distinguishing cooperative circumstances from the myriad of other problems and social interactions that people encounter-this is especially true when information about the payoffs to alternative behaviors or strategies is costly to acquire.

As I have mentioned, natural selection favors the evolution of conformist transmission because it helps to solve individual-level problems (Henrich and Boyd, 1998), and leads to an effective solution in many forms of social interaction, such as coordination games. Consequently, in order for natural selection to favor an ability to "switch-off" the conformist effect upon encountering a cooperation problem, individuals must be able to accurately distinguish cooperative dilemmas from all other dilemmas. To accomplish this, individuals must be able to acquire sufficient information about the relative payoffs received by individuals behaving in alternative ways. However, the amount of payoff information necessary to distinguish a cooperative dilemma from other problems (e.g., coordination interactions) is also sufficient to determine the optimal solution to the problem without any social learning. If people could acquire and process sufficient information to 'know' when to switch-off

\footnotetext{
16 Boyd et al. (2003) have combined the fact that the payoff difference between punishing and not-punishing is smaller than that between cooperating and not-cooperating with group selection to show that the conditions for the evolution of cooperation and punishment are much more plausible for cultural evolution than genetic evolution.
} 
conformist transmission, then they would be able to determine the optimal choice in the situation, and we would not expect to observe any social learning in social interactions. Nevertheless, when experimentalists have provided opportunities for social learning in economic interactions, subjects usually rely heavily on it (Kroll and Levy, 1992; Ball et al., 2001; Smith and Bell, 1994; Pingle, 1995; Pingle and Day, 1996; Offerman and Sonnemans, 1998; Wit, 1999). Smith and Bell (1994) have tested this explicitly by comparing subjects' strategic use of social information with their imitative use of this information, and found that subjects imitate others both when self-interest conflicts with group-interest and when they run in parallel. No difference is observed in the relative strengths of people's imitative tendencies (cf. Weinmann, 1994). ${ }^{17}$

Overall, given the complexity of human cultural adaptations and social environments as well as the lack of information about the costs and benefits of rarely observed alternative social behaviors, this constraint likely holds and is certainly more plausible than, for example, mutational restrictions that confine punishment-alleles and cooperation alleles to the same gene.

\subsection{Different processes of cultural group selection}

Once stable culturally transmitted differences arise between groups, at least three different forms of cultural group selection may influence the evolution of practices, beliefs, ideas and values: demographic swamping, intergroup competition and prestige-biased group selection. Demographic swamping produces changes in the frequency of cultural traits in an overall meta-population because some social groups (perhaps just one) reproduce new individuals faster than other groups as a consequence of some set of culturally transmitted ideas or practices that are relatively stable in those groups-this is natural selection acting on between-group cultural variation. Demographic swamping probably explains the spread of early agriculturalists into regions once dominated entirely by hunter-gatherers. Agriculturalists gradually replace foragers, increasingly compressing them into tracks of inarable land (Cavalli-Sforza et al., 1994; Young and Bettinger, 1992; Diamond, 1997). Such empirical cases of demographic swamping suggest that this is probably the slowest kind of cultural group selection, operating on time scales of millennia.

In intergroup competition, different cultural groups may also compete directly for access to resources through warfare and raiding. Cultural practices and beliefs that provide a competitive edge to groups in warfare will proliferate at the expense of traits that make groups less effective in competition (and more likely to be defeated, absorbed or dispersed). Such cultural traits might relate to beliefs about patrilocality, heroism, patriotism, economic cooperation (leading to surplus production), the villainy of foreigners, and the proper forms of social or political organization (or all of these). In exploring cultural group selection resulting from intergroup competition, Soltis et al. (1995) calculated evolutionary rates using a model based on group "extinctions" ("extinction" only implies that the group members

\footnotetext{
$\overline{17}$ Further, the availability of the payoff information required to distinguish between cooperative and other kinds of social interactions is often limited. If an individual enters the world and everyone is playing strategy "A", then he has no idea what will happen if he plays "B". If the underlying dilemma is cooperative and A is "cooperate", then everyone may receive the same payoffs, so it will 'look' like (be indistinguishable from) a coordination game.
} 
must be disbanded and scattered, not necessarily killed). Using empirical data from New Guinean horticultural groups, Soltis et al., estimated that a group-beneficial, cultural trait could spread to fixation on time scales of 500-1000 years.

One of the best-documented cases of cultural group selection occurred during the 18th century among the anthropologically famous ethnic groups of the Nuer and the Dinka. Before 1820, the Nuer and Dinka (Kelly, 1985) occupied adjacent regions in the southern Sudan. Despite inhabiting similar environments and possessing identical technology, the two groups differed in significant ways. Economically, both the Dinka and the Nuer raised cattle, but the Dinka maintained smaller herds of approximately nine cows per bull, while the Nuer maintained larger herds with two cows per bull. The Nuer ate mostly milk, corn and millet and rarely slaughtered cows, while the Dinka frequently ate beef. Politically, the Dinka lived in small groups, the largest of which corresponded to their wet season encampment. In contrast, the Nuer organized according to a patrilineal kin system that structured tribal membership across much larger geographic areas. Consequently, the size of a Dinka social group was limited by geography, whereas the Nuer system could organize much larger numbers of people over greater expanses of territory. Despite the similarity of their environments, these two groups showed substantial economic and political differences. Over about 100 years, starting in about 1820, the Nuer dramatically expanded their territory at the expense of the Dinka, who were driven off, killed, or captured and assimilated. As a result, Nuer beliefs and practices spread, fairly rapidly, across the landscape relative to Dinka beliefs and practices despite the fact that the Nuer were soon living in the once "Dinka environment".

Another subtler form of cultural group selection is likely to operate on even shorter time scales than intergroup competition: prestige-biased group selection. Under prestige-biased transmission, people preferentially copy individuals who get higher payoffs. The higher an individual's payoff, the more likely that individual is to be imitated. If individuals occasionally have opportunities to copy people in neighboring groups, then individuals from groups at more efficient (cooperative) equilibria will be preferentially imitated by individuals from groups at less efficient (non-cooperative) equilibrium because the average payoffs to individuals from cooperative groups are much higher than the average payoff of individuals in non-cooperative groups (Henrich and Boyd, 2001). Boyd and Richerson (2002b) have shown that, under a wide range of conditions, this form of cultural group selection will spread group-beneficial behaviors from a single group (at a group-beneficial equilibrium) through a meta-population of other groups that were previously stuck at more individualistic equilibria. Furthermore, this process allows more efficient equilibria (institutional forms) to recombine and spread. For example, in an ecology of many groups, one group may have drifted onto an efficient system of patrilineal inheritance (suppose this is more efficient than matrilineal or bilateral inheritance); another group may have evolved cooperative norms specifically for the construction of large buildings; and, a third group may have developed a segmentary lineage system that allows them to organize large numbers of warriors. The above process allows these three different kinds of behavioral equilibria to individually spread, and eventually recombine in a single social group, making this group the most successful/competitive group by far. Notably, this process is fast and can probably occur on time scales of decades or centuries. 


\section{Genes may respond to the changed social environment created by cultural evolution}

By systematically altering the selective environment faced by genes, cultural evolution via cultural group selection may lead to the subsequent spread of prosocial genes-genes that would not otherwise be favored without the action of cultural processes. As cultural group selection spreads punishment, prosocial genes (genetically encoded "preferences" for in-group altruism and punishment) may be favored if they allow individuals to avoid the costs of being punished for not cooperating. Because such prosocial genes would have evolved in a world with substantial amounts of culturally evolved, between-group variation, such genes would foster prosocial psychologies adapted to cue off local behavioral patterns. This would allow human social groups to vary in both their degree of prosociality and their domains of cooperation (house-building, warfare, fishing, etc.) in a manner not possible in non-cultural species. One way to think about these "preferences" is as learning biases that allow individuals to rapidly acquire prosocial behaviors, values or strategies by observing others, or with a minimum of punishment. Elsewhere, Henrich and Boyd (2001) have shown that prosocial genes will spread under a wide range of conditions in the altered environments produced by cultural group selection.

\section{Conclusions}

Besides providing a plausible evolutionary foundation for the empirically observed and experimentally measured patterns of human prosociality that cannot easily be accounted for by standard evolutionary models, the culture-gene coevolutionary approach solves two other problems in understanding cooperation and punishment that have not even been addressed by standard explanations.

Problem 1. If large-scale cooperation is a product of purely natural selection acting on genes to favor something such as indirect reciprocity, why is not large-scale cooperation more wide-spread in nature? Why do not other social species like baboons, sea lions, chimpanzees, and dolphins (which show evidence of kinship and maybe reciprocity) cooperate at human levels? The above approach, by rooting the development of large-scale cooperation in the details of human social learning, addresses this challenge. Other mammals do not cooperate to the degree humans do because they lack the social learning abilities that produce cultural evolution and behavioral equilibria not available to genetic transmission alone. Of course, this answer only pushes the question back to why more animals do not have human-style cultural capacities. In answering this question, Boyd and Richerson (1996) have shown that there is an adaptive valley in the evolution of cultural abilities that inhibits the spread of these abilities when rare. Thus, such cultural abilities should be rare in nature, but once a species crosses the 'cultural threshold', whole new evolutionary vistas open up (also see Henrich and Gil-White, 2001).

Problem 2. Unlike other animals, human cooperation varies in both scale and behavioral domains across social groups. As I mentioned, many social groups inhabit the same physical environment and possess the same technology, but cooperate to differing degrees and in different domains (e.g., the Nuer and Dinka). Standard evolutionary approaches struggle 
with these uniquely human observations-in other animals, cooperation and sociality do not vary much from group to group within a species. However, the coevolutionary approach suggests that rapid within-group cultural forces will drive different social groups to one of a myriad of different quasi-stable equilibria, where they may remain for long periods until the between-group component of cultural evolution sorts among the various equilibria. In selecting among alternative equilibria, cultural group selection processes may operate on much longer time scales than within-group processes, especially when alternative equilibria have similar effects on cultural group fitness. The rates of cultural evolution under group selection (decades, centuries and millennia) have about the right time scale to explain human history (Diamond, 1997), whereas genetic explanations are too slow and rational choice ones too fast.

\section{Acknowledgements}

My thanks goes to Natalie Smith, Robert Boyd, Pete Richerson, Ernst Fehr, Barkley Rosser, Dick Day, Steve Frank, the anonymous reviewer, Scott Atran, and members of the EHAP seminar at the University of Michigan for reviewing earlier versions of this paper. I would also like to express my gratitude to all the commentators for their excellent contributions.

\section{References}

Alexander, R., 1974. The evolution of social behavior. Annual Review of Ecology and Systematics 5, $325-383$. Alexander, R., 1987. The Biology of Moral Systems. Aldine De Gruyter, New York.

Aoki, K., 1982. A condition for group selection to prevail over counteracting individual selection. Evolution 36 (4), $832-842$.

Arnold, J., 1996. The archaeology of complex hunter-gatherers. Journal of Archaeological Method and Theory 3 (2), 77-126.

Asch, S.E., 1951. Effects of group pressure upon the modification and distortion of judgments. In: Guetzkow, H. (Ed.), Groups, Leadership and Men. Carnegie, Pittsburgh, pp. 177-190.

Axelrod, R., 1984. The Evolution of Cooperation. Basic Books, US.

Axelrod, R., 1986. An evolutionary approach to norms. American Political Science Review 80 (4), $1095-1111$.

Axelrod, R., Hamilton, W.D., 1981. The evolution of cooperation. Science 211, 1390-1396.

Axelrod, R., Dion, D., 1988. The further evolution of cooperation. Science 242, 1385-1389.

Ball, S., Eckel, C., Grossman, P., Zame, W., 2001. Status in markets. Quarterly Journal of Economics 155 (1), 161-181.

Baron, R., Vandello, J., Brunsman, B., 1996. The forgotten variable in conformity research: impact of task importance on social influence. Journal of Personality and Social Psychology 71 (5), 915-927.

Batson, D., Shaw, L., 1991. Evidence for alturism: toward a pluralism of prosocial motives. Psychological Inquiry 2 (2), 107-122.

Bendor, J., Mookherjee, D., 1987. Institutional structure and the logic of ongoing collective action. The American Political Science Review 81 (1), 129-154.

Ben-Ner, A., Putterman, L., 2000. Some implications of evolutionary psychology for the study of preferences and institutions. Journal of Economic Behavior and Organization 43 (1), 91-99.

Bliege Bird, R., Bird, D.W., 1997. Delayed reciprocity and tolerated theft: the behavioral ecology of food-sharing strategies. Current Anthropology 38 (1), 49-78.

Bolton, G.E., Katok, E., Zwick, R., 1998. Dictator game giving: rules of fairness versus acts of kindness. International Journal of Game Theory 27, 269-299. 
Boorman, S., Levitt, P., 1980. The Genetics of Altruism. Academic Press, New York.

Bowles, S., 2001. Individual interactions, group conflicts, and the evolution of preferences. In: Durlauf, S., Young, P. (Eds.), Social Dynamics. MIT Press, Cambridge, MA, pp. 155-190.

Boyd, R., 1988. Is the repeated prisoner's dilemma a good model of reciprocal altruism? Ethology and Sociobiology 9, 211-222.

Boyd, R., 1989. Mistakes allow evolutionary stability in the repeated prisoner's dilemma game. Journal of Theoretical Biology 136, 47-56.

Boyd, R., Lorderbaum, J.P., 1987. No pure strategy is evolutionarily stable in the repeated Prisoner's dilemma game. Nature 32 (6117), 58-59.

Boyd, R., Richerson, P.J., 1985. Culture and the Evolutionary Process. University of Chicago Press, Chicago, IL.

Boyd, R., Richerson, P.J., 1987. The evolution of ethnic markers. Cultural Anthropology 2 (1), 27-38.

Boyd, R., Richerson, P.J., 1988. The evolution of reciprocity in sizable groups. Journal of Theoretical Biology 132, 337-356.

Boyd, R., Richerson, P.J., 1989. The evolution of indirect reciprocity. Social Networks 11 (3), 213-236.

Boyd, R., Richerson, P.J., 1990. Group selection among alternative evolutionarily stable strategies. Journal of Theoretical Biology 145, 331-342.

Boyd, R., Richerson, P.J., 1992. Punishment allows the evolution of cooperation (or anything else) in sizable groups. Ethology and Sociobiology 13 (3), 171-195.

Boyd, R., Richerson, P., 1996. Why culture is common, but cultural evolution is rare. Proceedings of the British Academy 88, 77-93.

Boyd, R., Richerson, P.J., 2000. Memes: universal acid or a better mouse trap. In: Aunger, R. (Ed.), Darwinizing Culture: The Status of Memetics as a Science. Oxford University Press, Oxford, pp. 143-162.

Boyd, R., Richerson, P., 2002a. Group beneficial norms can spread rapidly in a structured population. Journal of Theoretical Biology 215, 287-296.

Boyd, R., Richerson, P., 2002b. Solving the puzzle of human cooperation. In: Levinson, S. (Ed.), Evolution and Culture. MIT Press, Cambridge, MA.

Boyd, R., Silk, J., 1997. How Humans Evolved. W.W. Norton \& Company, New York.

Boyd, R., Gintis, H., Bowles, S., Richerson, P.J., 2003. The evolution of altruistic punishment. PNAS 100 (6), 3531-3535.

Brown, R., Armelagos, G., 2001. Apportionment of racial diversity: a review. Evolutionary Anthropology 10, 34-40.

Brown, W.M., Moore, C., 2002. Smile asymmetries and reputation as reliable indicators of likelihood to cooperate: an evolutionary analysis. Advances in Psychological Research 11, 59-78.

Cavalli-Sforza, L.L., Menozzi, P., Piazza, A., 1994. The History and Geography of Human Genes. Princeton University Press, Princeton, NJ.

Crow, J., Aoki, K., 1982. Group selection for a polygenic behavioral trait: a differential proliferation model. Proceedings of the National Academy of Sciences of the United States of America 79 (8), 2628-2631.

Daly, M., Wilson, M., 1988. Homicide. Aldine de Gruyter, New York.

Davis, D.D., Holt, C.A., 1993. Experimental Economics. Princeton University Press, Princeton, NJ.

Dawkins, R., 1976. The Selfish Gene. Oxford University Press, Oxford.

Dawkins, R., 1982. The Extended Phenotype. Oxford University Press, Oxford.

Diamond, J.M., 1997. Guns, Germs, and Steel: The Fates of Human Societies. W.W. Norton \& Company, New York.

Ekman, P., 1992. Telling Lies. Norton, New York.

Ekman, P., O’Sullivan, M., 1991. Who can catch a liar? American Psychologist 46, 913-920.

Ellison, G., Fudenberg, D., 1993. Rules of thumb for social learning. Journal of Political Economy 101 (4), 612-643.

Fehr, E., Gächter, S., 2000. Cooperation and punishment in public goods experiments. American Economic Review 90 (4), 980-995.

Fehr, E., Gächter, S., 2002. Altruistic punishment in humans. Nature 415, 137-140.

Fehr, E., Henrich, J. (forthcoming). Is strong reciprocity a maladaption? In: Hammerstein, P. (Ed.), Genetic and Cultural Evolution of Cooperation. MIT Press, Cambridge, MA.

Fehr, E., Fischbacher, U., Gächter, S., 2002. Strong reciprocity, human cooperation and the enforcement of social norms. Human Nature 13, 1-25. 
Fletcher, D.J.C., Michener, C.D., 1987. Kin Recognition in Animals. Wiley, New York.

Frank, R., 1988. Passions within Reason: The Strategic Role of the Emotions. W.W. Norton \& Company, New York.

Frank, S., 1995. George price's contributions to evolutionary genetics. Journal of Theoretical Biology 175, 373388.

Frank, S.A., 1998. Foundations of Social Evolution. Princeton University Press, Princeton, NJ.

Frank, R., Gilovich, T., Regan, D.T., 1993. The evolution of one-shot cooperation: an experiment. Ethology and Sociobiology 14, 247-256.

Fudenberg, D., Maskin, E., 1986. The folk theorem in repeated games with discounting or with incomplete information. Econometrica 54, 533-556.

Furnham, A., 1984. Studies of cross-cultural conformity: a brief and critical review. Psychologia: An International Journal of Psychology in the Orient 27 (1), 65-72.

Gintis, H., 2000. Strong reciprocity and human sociality. Journal of Theoretical Biology 206, 169-179.

Gintis, H., Smith, E.A., Bowles, S., 2001. Costly signaling and cooperation. Journal of Theoretical Biology 213 (1), 103-119.

Hamilton, W.D., 1964. The genetical evolution of social behaviour. Journal of Theoretical Biology 7, 1-16.

Hamilton, W.D., 1972. Altruism and related phenomena, mainly in the social insects. Annual Review of Ecology and Systematics 3, 192-232.

Hamilton, W.D., 1975. Innate social aptitudes of man: an approach from evolutionary genetics. In: Fox, R. (Ed.), Biosocial Anthropology. Malaby Press, London, pp. 133-156.

Harris, M., 1977. Cannibals and Kings: The Origins of Cultures. Random House, New York.

Harris, M., 1979. Cultural Materialism: The Struggle for a Science of Culture. Random House, New York.

Harris, J.R., 1998. The Nurture Assumption: Why Children Turn Out the Way They Do. Touchstone, New York.

Hartl, D.L., Clark, A.G., 1989. Principles of Population Genetics. Sinauer Associates, Sunderland, MA.

Hayek, F., 1979. The political order of a free people. Law, Legislation and Liberty, vol. 3. University of Chicago Press, Chicago.

Henrich, J., 2001. Cultural transmission and the diffusion of innovations: adoption dynamics indicate that biased cultural transmission is the predominate force in behavioral change and much of sociocultural evolution. American Anthropologist 103, 992-1013.

Henrich, J., Boyd, R., 1998. The evolution of conformist transmission and the emergence of between-group differences. Evolution and Human Behavior 19, 215-242.

Henrich, J., Boyd, R., 2001. Why people punish defectors: weak conformist transmission can stabilize costly enforcement of norms in cooperative dilemmas. Journal of Theoretical Biology 208, 79-89.

Henrich, J., Boyd, R., 2002. On modeling cultural evolution: why replicators are not necessary for cultural evolution. Journal of Cognition and Culture 2 (2), 87-112.

Henrich, J., Gil-White, F., 2001. The evolution of prestige: freely conferred deference as a mechanism for enhancing the benefits of cultural transmission. Evolution and Human Behavior 22 (3), 165-196.

Henrich, J., Smith, N., in press. Comparative experimental evidence from Peru, Chile and the US shows substantial variation among social groups. In: Henrich, J., Boyd, R., Bowles, S., Gintis, H., Fehr, E., Camerer, C. (Eds.), Cooperation, Punishment and Self Interest: Experimental and Ethnographic Evidence from 15 Small Scale Societies. Oxford University Press, New York, and University of Michigan.

Henrich, J., Boyd, R., Bowles, S., Gintis, H., Camerer, C., Fehr, E., McElreath, R., 2001. In search of Homo economicus: experiments in 15 small-scale societies. American Economic Review 91, 73-78.

Hirshleifer, J., 1982. Evolutionary models in economics and law: cooperation vs. conflict strategies. Research in Law and Economics 4, 1-60.

Hirshleifer, J., Martinez, C., 1988. What strategies can support the evolutionary emergence of cooperation. Journal of Conflict Resolution 32 (2), 367-398.

Hirshleifer, D., Rasmusen, E., 1989. Cooperation in a repeated prisoner's dilemma with ostracism. Journal of Economic Behavior and Organization 12, 87-106.

Hoffman, E., McCabe, K., Smith, V., 1998. Behavioral foundations of reciprocity: experimental economics and evolutionary psychology. Economic Inquiry 36, 335-352.

Insko, C.A., Smith, R.H., Alicke, M.D., Wade, J., Taylor, S., 1985. Conformity and group size: the concern with being right and the concern with being liked. Personality and Social Psychology Bulletin 11 (1), 41-50. 
Johnson, A., 2003. Families of the Forest: Matsigenka Indians of the Peruvian Amazon. University of California, Berkeley, CA.

Johnson, A., Earle, T., 1987. The Evolution of Human Societies: From Foraging Group to Agrarian State. Stanford University Press, Stanford.

Joshi, N.V., 1987. Evolution of cooperation by reciprocation within structured demes. Journal of Genetics 66 (1), 69-84.

Kagel, J.H., Roth, A.E., 1995. The Handbook of Experimental Economics. Princeton University Press, Princeton, NJ.

Keller, M., Edelstein, W., Schmid, C., Fang, G., Fang, F.-X., 1998. Reasoning about relationships and obligations in close relationships: a comparison across two cultures. Developmental Psychology 34 (4), 731-741.

Kelly, R.C., 1985. The Nuer Conquest. University of Michigan Press, Ann Arbor.

Kelly, J.K., 1992. Restricted migration and the evolution of altruism. Evolution 46 (5), 1492-1495.

Klein, R.G., 1989. The Human Career: Human Biological and Cultural Origins. University of Chicago, Chicago.

Kroll, Y., Levy, H., 1992. Further tests of the separation theorem and the capital asset pricing model. American Economic Review 82 (3), 664-670.

Ledyard, J.O., 1995. Public goods: a survey of experimental research. The Handbook of Experimental Economics. Princeton University Press, Princeton, NJ, pp. 111-194.

Lee, R.B., 1979. The !Kung San: Men, Women, and Work in a Foraging Society. Cambridge University Press, Cambridge.

Leimar, O., Hammerstein, P., 2001. Evolution of cooperation through indirect reciprocity. Proceedings: Biological Sciences 268 (1468), 745-753.

Lorberbaum, J., 1994. No strategy is evolutionarily stable in the repeated prisoner's dilemma. Journal of Theoretical Biology 168, 117-130.

Lorberbaum, J.P., Bohning, D.E., Shastri, A., Sine, L., 2002. Are there really no evolutionary stable strategies in the iterated prisoner's dilemma. Journal of Theoretical Biology 214, 155-169.

Low, B.S., 2000. Why Sex Matters: A Darwinian Look at Human Behavior. Princeton University Press, Princeton, NJ.

Maynard Smith, J., 1998. The origin of altruism. Nature 393 (18), 639-640.

McAdams, R.H., 1998. The origin, development and regulation of norms. Michigan Law Review 96, 338.

McCabe, K., Smith, V., LePore, M., 2000. Intentionality detection and "mindreading": why does game form matter? Proceedings of the National Academy of Science 97 (8), 4404-4409.

McElreath, R., Boyd, R., Richerson, P., 2003. Shared norms can lead to the evolution of ethnic markers. Current Anthropology 44 (1), 123-129.

Neto, F., 1995. Conformity and independence revisited. Social Behavior and Personality 23 (3), 217-222.

Nowak, M.A., Sigmund, K., 1998. Evolution of indirect reciprocity by image scoring. Nature 393 (6685), $573-577$.

Ockenfels, A., Selten, R., 2000. An experiment on the hypothesis of involuntary truth-signaling in bargaining. Games and Economic Behavior 33 (1), 90-116.

Offerman, T., Sonnemans, J., 1998. Learning by experience and learning by imitating others. Journal of Economic Behavior and Organization 34 (4), 559-575.

Patton, J.Q., 2000. Reciprocal altruism and warfare: a case from the Ecuadorian Amazon. In: Cronk, L., Chagnon, N., Irons, W. (Eds.), Adaption and Human Behavior: An Anthropological Perspective. Aldine de Gruyter, New York, pp. 417-436.

Pingle, M., 1995. Imitation vs. rationality: an experimental perspective on decision-making. Journal of Socio-economics 24, 281-315.

Pingle, M., Day, R.H., 1996. Modes of economizing behavior: experimental evidence. Journal of Economic Behavior and Organization 29, 191-209.

Plomin, R., Defries, J., McLearn, G. E., 2000. Behavioral Genetics. Freeman, New York.

Price, G., 1970. Selection and covariance. Nature 227, 520-521.

Price, G., 1972. Extensions of covariance selection mathematics. Annals of Human Genetics 35, 485-490.

Queller, D.C., 1992a. A general model for kin selection. Evolution 46 (2), 376-380.

Queller, D., 1992b. Quantitative genetics, inclusive fitness, and group selection. The American Naturalist 139 (3), 541-558.

Rappaport, R.A., 1984. Pigs for the Ancestors. Yale University Press, New Haven. 
Richerson, P., Boyd, R., 1998. The evolution of ultrasociality. In: Eibl-Eibesfeldt, I., Salter, F.K. (Eds.), Indoctrinability, Ideology and Warfare. Berghahn Books, New York, pp. 71-96.

Richerson, P., Boyd, R., 2000. Complex societies: the evolutionary dynamics of a crude superorganism. Human Nature 10, 253-289.

Ridley, M., 1993. The Red Queen: Sex and the Evolution of Human Nature. Penguin Books, New York.

Rogers, A., 1990. Group selection by selective emigration: the effects of migration and kin structure. The American Naturalist 135 (3), 339-413.

Rogers, E.M., 1995. Diffusion of Innovations. Free Press, New York.

Roscoe, P., 2002. The hunters and gatherers of new guinea. Current Anthropology 43 (1), 153-162.

Rosekrans, M.A., 1967. Imitation in children as a function of perceived similarity to a social model and vicarious reinforcement. Journal of Personality and Social Psychology 7 (3), 307-315.

Roth, A.E., Prasnikar, V., Okuno-Fujiwara, M., Zamir, S., 1991. Bargaining and market behavior in Jerusalem, Ljubljana, Pittsburgh and Tokyo: an experimental study, Pittsburgh and Tokyo: an experimental study. American Economic Review 81 (5), 1068-1095.

Sepher, J., 1983. Incest, the Biosocial View. Academic Press, New York.

Smith, J.M., Bell, P.A., 1994. Conformity as a determinant of behavior in a resource dilemma. Journal of Social Psychology 134 (2), 191-200.

Sober, E., Wilson, D.S., 1998. Unto Others: The Evolution and Psychology of Unselfish Behavior. Harvard University Press, Cambridge.

Soltis, J., Boyd, R., Richerson, P.J., 1995. Can group-functional behaviors evolve by cultural group selection? An empirical test. Current Anthropology 36 (3), 473-494.

Spencer, H., 1891. Essays: Scientific, Political, and Speculative. Williams \& Northgate, London.

Stotland, E., Dunn, R., 1962. Identification, "oppositeness," authoritarianism, self-esteem, and birth order. Psychological Monographs: General and Applied 76 (9), 1-30.

Stotland, E., Dunn, R., 1963. Empathy, self-esteem and birth order. Journal of Abnormal and Social Psychology $66(6), 532-540$.

Tomasello, M., 1994. The question of chimpanzee culture. In: Wrangham, R., McGrew, W.C., de Waal, F.B.M., Heltne, P.G. (Eds.), Chimpanzee Cultures. Harvard University Press, Cambridge, MA, pp. 301-317.

Tomasello, M., 2000. The Origins of Human Cognition. Harvard University Press, Cambridge.

Tooby, J., Cosmides, L., 1989. Evolutionary psychology and the generation of culture: theoretical considerations. Ethology and Sociobiology 10, 29-49.

Trivers, R.L., 1971. The evolution of reciprocal alturism. The Quarterly Review of Biology 46, 34-57.

Turner, J., Maryanski, A., 1979. Functionalism. Benjamin/Cummings, Menlo Park, CA.

Vayda, A.P., 1971. Phases of the process of war and peace among the Marings of new Guinea. Oceania 42, 1-24.

Wade, M., 1985. Soft selection, hard selection, kin selection, and group selection. The American Naturalist 125 (1), 61-73.

Wedekind, C., Milinski, M., 2000. Cooperation through image scoring in humans. Science 288, 850-852.

Weinmann, J., 1994. Individual behavior in a free riding experiment. Journal of Public Economics 54 (2), $185-200$.

Westermarck, E., 1894. The History of Human Marriage. Macmillan, London.

Wiessner, P., 1983. Style and social information in kalahari san projectile points. American Antiquity 48 (2), $253-275$.

Williams, G., 1966. Adaption and Natural Selection: A Critique of Some Current Evolutionary Theory. Princeton University Press, Princeton, NJ.

Wilson, D.S., Dugatkin, L., 1997. Group selection and assortative interactions. The American Naturalist 149 (2), 336-351.

Wit, J., 1999. Social learning in a common interest voting game. Games and Economic Behavior 26, 131-156.

Wolf, A.P., 1970. Childhood association and sexual attraction: a further test of the Westermarck hypothesis. American Anthropologists 72, 503-515.

Young, D., Bettinger, R.L., 1992. The numic spread: a computer simulation. American Antiquity 57 (1), 85-99. 